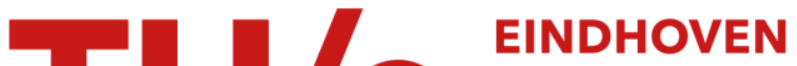

\section{Effective dispersion equations for reactive flows involving free boundaries at the microscale}

\section{Citation for published version (APA):}

Kumar, K., Noorden, van, T. L., \& Pop, I. S. (2011). Effective dispersion equations for reactive flows involving free boundaries at the microscale. Multiscale Modeling \& Simulation, 9(1), 29-58.

https://doi.org/10.1137/100804553

DOI:

$10.1137 / 100804553$

Document status and date:

Published: 01/01/2011

\section{Document Version:}

Publisher's PDF, also known as Version of Record (includes final page, issue and volume numbers)

\section{Please check the document version of this publication:}

- A submitted manuscript is the version of the article upon submission and before peer-review. There can be important differences between the submitted version and the official published version of record. People interested in the research are advised to contact the author for the final version of the publication, or visit the $\mathrm{DOI}$ to the publisher's website.

- The final author version and the galley proof are versions of the publication after peer review.

- The final published version features the final layout of the paper including the volume, issue and page numbers.

Link to publication

\section{General rights}

Copyright and moral rights for the publications made accessible in the public portal are retained by the authors and/or other copyright owners and it is a condition of accessing publications that users recognise and abide by the legal requirements associated with these rights.

- Users may download and print one copy of any publication from the public portal for the purpose of private study or research.

- You may not further distribute the material or use it for any profit-making activity or commercial gain

- You may freely distribute the URL identifying the publication in the public portal.

If the publication is distributed under the terms of Article 25fa of the Dutch Copyright Act, indicated by the "Taverne" license above, please follow below link for the End User Agreement:

www.tue.nl/taverne

Take down policy

If you believe that this document breaches copyright please contact us at:

openaccess@tue.nl

providing details and we will investigate your claim. 


\title{
EFFECTIVE DISPERSION EQUATIONS FOR REACTIVE FLOWS INVOLVING FREE BOUNDARIES AT THE MICROSCALE*
}

\author{
K. KUMAR ${ }^{\dagger}$, T. L. VAN NOORDEN ${ }^{\dagger}$, AND I. S. $\mathrm{POP}^{\dagger}$
}

\begin{abstract}
We consider a pore-scale model for reactive flow in a thin two-dimensional strip, where the convective transport dominates the diffusion. Reactions take place at the lateral boundaries of the strip (the walls), where the reaction product can deposit in a layer with a nonnegligible thickness compared to the width of the strip. This leads to a free boundary problem, in which the moving interface between the fluid and the deposited (solid) layer is explicitly taken into account. Using asymptotic expansion methods, we derive an upscaled, one-dimensional model by averaging in the transversal direction. The result is consistent with (Taylor dispersion) models obtained previously for a constant geometry. Finally, numerical computations are presented to compare the outcome of the effective (upscaled) model with the transversally averaged, two-dimensional solution.
\end{abstract}

Key words. porous media, upscaling, reactive flow, free boundary problem, Taylor dispersion

AMS subject classifications. 35B27, 76S05, 35K57

DOI. $10.1137 / 100804553$

1. Introduction. We consider a pore-scale model for reactive flows in porous media. A fluid flowing through the void space of the medium (the pores) transports some dissolved ions. Reactions can take place at the pore walls, with the resulting component being attached to or detached from the pore walls. Two situations can be identified in this case. In the first situation, we assume that the reaction product forms a very thin layer that does not influence the pore space and that the pore-scale models are written in a fixed geometry. Alternatively, one assumes that the thickness of the deposited layer is not negligible when compared to the pore thickness, particularly when thin pores are considered. Then the reactions can lead to variations in the pore space and hence in the flow domain. The interface separating this domain from the the solid part is a free boundary having an unknown, time dependent location. In this paper, we consider the case with variable pore space.

The scenario described above is generic. It can be encountered, for example, in crystal precipitation and dissolution (see, e.g., $[9,11,24,26,25]$ ), atomic layer deposition [17], chemical vapor deposition [28] and etching in a heterogenous surface [33, 34], concrete carbonation [21,22] and cement hydration [5], or biological applications such as biofilm growth [27] and thrombosis [35].

In practice one is often not interested in the detailed solution on microscale but only in the description of the average behavior of the system. In such situations, upscaled models are very useful as they describe average behavior with relatively low computational efforts. Considering the two-dimensional (2-D) strip as a representative pore-scale geometry, by the upscaled or effective model, we mean the set of equations defined in one-dimension, the solution of which describes the average behavior of the thin strip. These effective equations clearly depend upon the choice of microscopic

*Received by the editors August 5, 2010; accepted for publication (in revised form) October 6, 2010; published electronically January 20, 2011.

http://www.siam.org/journals/mms/9-1/80455.html

${ }^{\dagger}$ Centre for Analysis, Scientific Computing and Applications, Technische Universiteit Eindhoven, P.O. Box 513, 5600 MB Eindhoven, The Netherlands (k.kumar@tue.nl, t.l.v.noorden@tue.nl, i.pop@tue.nl). The work of K. Kumar was supported by the Dutch Technology Foundation STW, project 07796 . 
model. Moreover, the effective model also depends on the ratio of the time scales for the diffusion and the convective transport, referred to as the Péclet number Pe. For the case of moderate or small $\mathbf{P e}$, when diffusion dominates or is in balance with the transport, the situation is well understood in both fixed and variable geometry frameworks (see, e.g., [7, 9, 11, 12, 13, 14, 15, 23, 24, 25, 26, 29] and references therein). The present work builds on the the results in [25], where a model for crystal dissolution and precipitation involving free boundaries is considered in a thin strip but for moderate $\mathbf{P e}$.

Here we consider the case of high Peclét number, $\mathbf{P e} \gg 1$. Then the convective transport dominates the diffusion, and it is observed that the net diffusion is enhanced by the transport term itself, leading to Taylor dispersion [32]. In the fixed geometry case, the Taylor dispersion mechanism is investigated in $[2,4,31]$. In the same context, reactive flow under dominating convective transport or reaction is studied in $[1,6,10,18,20,30,36]$, presenting either a formal derivation of dispersion models or rigorous convergence proofs for the upscaling procedure. In a similar context, in [19] an upscaled model of hyperbolic type is derived, sustained by rigorous mathematical arguments. In this paper, we derive upscaled equations for the convection-diffusionreaction system for the thin strip, taking into account the changes in the geometry of the microscale in the transport dominated regime. Because of the changes in the geometry, the flow profile does not remain constant, which is in contrast to the Taylor dispersion models for the fixed geometry case [10]. As in [10, 25], we employ the asymptotic expansion method to derive the upscaled flow and concentration equations and, in particular, the Taylor dispersion terms. It is worth noting that restricting only to the leading order terms leads to an upscaled model of hyperbolic type, and dispersion terms are lost. Therefore we also take into account the first order correctors and avoid making a specific choice of the integration constants, as in the anisotropic singular perturbation approach (see $[31,10]$ ). The advantage of such an upscaling is evidenced by the numerical experiments. Figure 1 shows the comparison of the present upscaled model named Upscaled with the other simpler macroscopic models and clearly the upscaled model derived here provides more accurate description of the average behavior given by the name 2-D Average. We discuss the details of these numerical experiments and other macroscopic equations in section 4 .

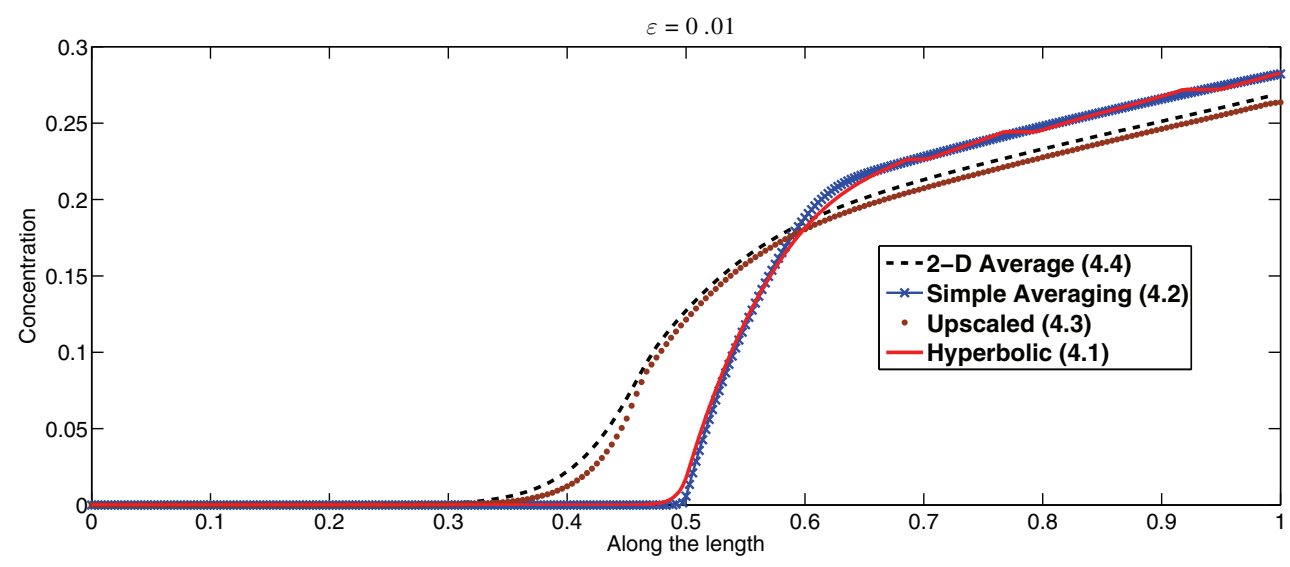

FIG. 1. Concentration profile using different upscaled models. 
The paper is organized as follows. The modeling details are provided in section 2 , followed by the derivation of the effective model in the case of a variable geometry in section 3. Section 4 provides numerical experiments, including comparisons with other upscaled equations, whereas Appendix A gives a derivation, using a different approach, of the upscaled model for the fixed geometry case.

2. The mathematical model. We start with the modeling for the 2-D thin strip of length $L[\mathrm{~m}]$ and width $\ell[\mathrm{m}]$. The thin strip is represented by

$$
\mathcal{Y}:=(0, L) \times(-\ell, \ell)
$$

The boundary of the strip consists of three parts: the solid part defined by

$$
\Gamma_{s}:=(0, L) \times\{ \pm \ell\}
$$

the inflow part defined by

$$
\Gamma_{i}:=\{0\} \times(-\ell, \ell),
$$

and the outflow part defined by

$$
\Gamma_{o}:=\{L\} \times(-\ell, \ell) .
$$

As described above, because of the reactions (attachment/detachment) taking place at the solid boundaries of the strip, the thickness of the layer attached to the boundary may change with time. The thickness of this layer is denoted by $d(x, t)[\mathrm{m}]$. For simplicity, we assume that initially the layer thickness on both the upper and the lower parts of $\Gamma_{s}$ is equal. This implies symmetry with respect to the x-axis. Note that for $d=\ell$, the thin strip will be blocked, leading to clogging. Separate models are required to treat this case. Thus, throughout this paper, to rule out clogging we assume that $d(x, t)<\ell$.

Because of the growth of the layers, the pore structure changes, leading to a change in the flow. We include this effect of change in flow due to the changes in geometry. The region occupied by the fluid is represented by

$$
\Omega(t):=\left\{(x, y) \in \mathbb{R}^{2} \mid 0 \leq x \leq L,-(\ell-d(x, t)) \leq y \leq(\ell-d(x, t))\right\} .
$$

The boundary of $\Omega(t)$ contains three parts: the lateral boundary $\Gamma_{g}(t)$, denoting the interface between the fluid phase and the deposited layer, defined by

$$
\Gamma_{g}(t):=\left\{(x, y) \in \mathbb{R}^{2} \mid 0 \leq x \leq L, y \in\{-(\ell-d(x, t)),(\ell-d(x, t))\}\right\} ;
$$

$\Gamma_{i f}(t)$, the inflow boundary at $x=0$ for the flow of the fluid phase,

$$
\Gamma_{i f}(t):=\left\{(x, y) \in \mathbb{R}^{2} \mid x=0,-(\ell-d(0, t)) \leq y \leq(\ell-d(0, t))\right\} ;
$$

and $\Gamma_{o f}(t)$, the outflow boundary at $x=L$ for the fluid phase,

$$
\Gamma_{o f}(t):=\left\{(x, y) \in \mathbb{R}^{2} \mid x=L,-(\ell-d(L, t)) \leq y \leq(\ell-d(L, t))\right\} .
$$

Note that $\Gamma_{i f} \subset \Gamma_{i}, \Gamma_{o f} \subset \Gamma_{o}$, but $\Gamma_{g}$ and $\Gamma_{s}$ need not have common points. A sketch of the geometry is shown in Figure 2. 


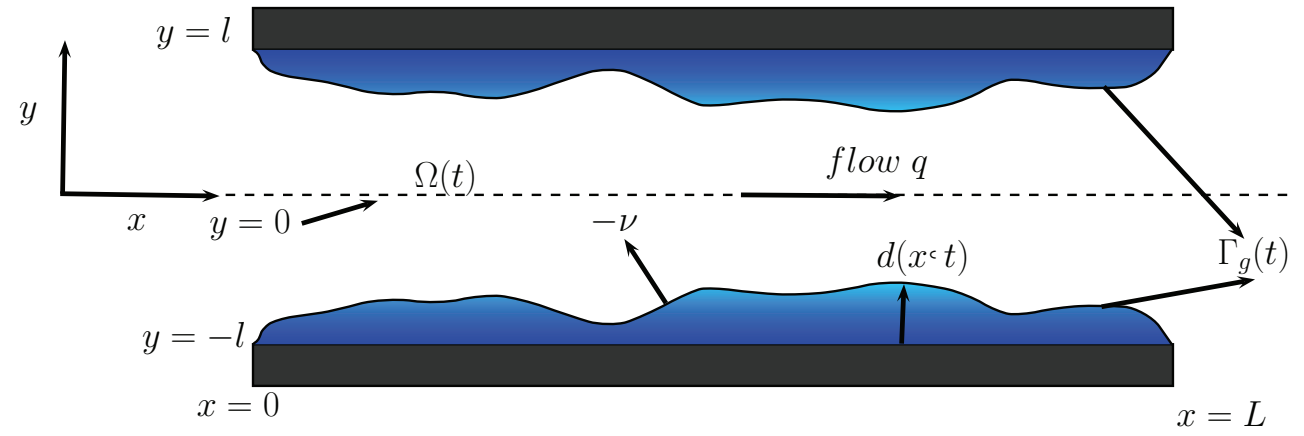

FIG. 2. Reactive flow in 2-D thin strip (pore geometry); $y=0$ is the line of symmetry.

For the solutes in the thin strip, the different processes are diffusion, transport by the fluid flow, and reactions taking place at the boundaries of the strip. We denote the concentration of the solute by $u\left[\mathrm{~mol} / \mathrm{m}^{3}\right]$. The convection-diffusion equation describing the transport process of the solute concentration is

$$
\begin{aligned}
\partial_{t} u & =\nabla \cdot(D \nabla u-q u), \\
u & =u_{b} \quad \text { on } \Gamma_{i}(t), \\
\partial_{x} u & =0 \quad \text { on } \Gamma_{o}(t),
\end{aligned}
$$

where $u_{b}\left[\mathrm{~mol} / \mathrm{m}^{3}\right]$ is a nonnegative constant, $D\left[\mathrm{~m}^{2} / \mathrm{s}\right]$ is the diffusion coefficient, and $q[\mathrm{~m} / \mathrm{s}]$ is the flow field.

We assume that reactions such as precipitation and dissolution take place only at the interface between the fluid and the solid, that is, $\Gamma_{g}(t)$. The corresponding mathematical description involves the outer normal $\nu$. At the lower part of $\Gamma_{g}(t)$, this is

$$
\nu=\left(\partial_{x} d,-1\right)^{T} / \sqrt{1+\left(\partial_{x} d\right)^{2}} .
$$

At the lower part of $\Gamma_{g}(t)$, mass conservation yields

$$
\nu \cdot(D \nabla u-q u)=v_{n}(\rho-u),
$$

where $\rho\left[\mathrm{mol} / \mathrm{m}^{3}\right]$ is the molar density of the solute in the solid phase (the adsorbed substance, or the precipitate) and $v_{n}$ is the outward normal velocity of the interface. Furthermore, the normal velocity of the interface $v_{n}[\mathrm{~m} / \mathrm{s}]$ is proportional to the reaction rate $f=f(u, \rho d)$,

$$
\rho v_{n}=-f(u, \rho d) .
$$

Here $f$ is a given function assumed to be sufficiently smooth. Similar results can be derived formally even for non-Lipschitz rates like Freundlich isotherms or multivalued reaction rates as encountered for dissolution processes. Such rates are used in the numerical computations presented in section 4 .

The velocity of a point $G(t)=(x(t),-\ell+d(x(t), t))$ on the lower part of the boundary $\Gamma_{g}(t)$ is given by

$$
G^{\prime}(t)=\left(x^{\prime}(t), \partial_{x} d(x(t), t) x^{\prime}(t)+\partial_{t} d(x(t), t)\right) .
$$


Then the normal velocity $v_{n}$ becomes

$$
v_{n}=\nu \cdot G^{\prime}(t)=-\frac{\partial_{t} d}{\sqrt{1+\left(\partial_{x} d\right)^{2}}},
$$

and (2.4) transforms into

$$
\rho \partial_{t} d=f(u, \rho d) \sqrt{1+\left(\partial_{x} d\right)^{2}} .
$$

The flow problem is modeled by the Stokes equations,

$$
\begin{aligned}
\mu \triangle q & =\nabla p, \\
\nabla \cdot q & =0,
\end{aligned}
$$

where $p[\mathrm{~Pa}]$ is the pressure field and $\mu[\mathrm{Pa}-\mathrm{s}]$ is the dynamic viscosity. At the inlet, we take a parabolic velocity profile normal to the inlet,

$$
q(0, y, t)=Q\left((\ell-d(0, t))^{2}-y^{2}\right) e_{1},
$$

where $Q>0$ is a normalization factor related to the total flow in the x-direction and $e_{1}$ is the unit vector along the x-axis. For the outlet, we prescribe the pressure $p=0$.

2.1. The dimensionless form. Before seeking an effective model, we bring (2.1)-(2.8) to a nondimensional form. We introduce therefore the reference time $t_{r e f}:=T$, coordinates $\left(x_{r e f}, y_{r e f}\right):=(L, \ell)$, velocity $q_{r e f}:=Q$, pressure $p_{r e f}$, and concentration $u_{r e f}$. The reference time is the characteristic convective transport time, satisfying

$$
T=\frac{L}{Q}
$$

We consider the case of thin strips, characterized by the ratio of its width to the length, $\varepsilon:=\ell / L$, and are interested in the limiting case $\varepsilon \searrow 0$. By an abuse of notation, we define the dimensionless independent variables and parameters

$$
x:=x / L, \quad y:=y / \ell, \quad t:=t / T, \quad \rho=\rho / u_{r e f}
$$

and the $\varepsilon$-dependent dimensionless quantities

$$
\begin{aligned}
& u^{\varepsilon}:=u / u_{\text {ref }}, \quad d^{\varepsilon}:=d / \ell, \quad q^{\varepsilon}:=q / Q, \\
& P^{\varepsilon}:=p / p_{\text {ref }}, \quad D:=\frac{D T}{\varepsilon^{-\alpha} L^{2}}, \quad \mu:=\frac{\mu L q_{r e f}}{\ell^{2} p_{\text {ref }}} .
\end{aligned}
$$

Remark 2.1. To understand the particular scaling in the dimensionless $D$, we note that the original diffusion coefficient $D$ and the length scale $L$ define a diffusion time scale $T_{D}:=L^{2} / D$. Here we are interested in the convection dominated regime; thus $T_{D} \gg T$. The ratio of these times is commonly defined as the Péclet number, for which we assume

$$
\mathbf{P e}=\frac{T_{D}}{T}=\varepsilon^{-\alpha}
$$

for some $\alpha>0$. We consider only the case $0<\alpha<2$, which corresponds to the Taylor dispersion regime. Note that applying this strategy for the experiments carried out 
by Taylor [32], the exponent $\alpha$ is close to 1.6 and to 1.9 (see also [10]). The case $\alpha<0$ corresponds to a diffusion dominated flow, and there will be no gradient in the concentration in the vertical direction. The case $\alpha=0$ has been treated in [25]. Whenever $\alpha \geq 2$, the gradient of the concentration along the width becomes too large, and other upscaling approaches should be considered.

Remark 2.2. The chemical processes such as adsorption, desorption, deposition, or precipitation and dissolution define a characteristic reaction time scale, $T_{R}$. This can be interpreted as the time needed to deposit a layer of thickness $\ell$. Then one can define the Damköhler number as the ratio of $T_{R}$ and $T$. Here we are interested in the regime of a moderate Damköhler number, thus when $T_{R} \approx T$. In view of the scaling, the derivatives become

$$
\partial_{x} \mapsto \frac{1}{L} \partial_{x}, \quad \partial_{y} \mapsto \frac{1}{\varepsilon L} \partial_{y}, \quad \partial_{t} \mapsto \frac{1}{T} \partial_{t},
$$

and the geometry is scaled as

$$
\Omega^{\varepsilon}(t):=\left\{(x, y) \in \mathbb{R}^{2} \mid 0 \leq x \leq 1,-\left(1-d^{\varepsilon}(x, t)\right) \leq y \leq\left(1-d^{\varepsilon}(x, t)\right)\right\} .
$$

The boundaries of $\Omega^{\varepsilon}(t)$ are then defined by the lateral boundary $\Gamma_{g}^{\varepsilon}(t)$

$$
\Gamma_{g}^{\varepsilon}(t):=\left\{(x, y) \in \mathbb{R}^{2} \mid 0 \leq x \leq 1, y \in\left\{-\left(1-d^{\varepsilon}(x, t)\right),\left(1-d^{\varepsilon}(x, t)\right)\right\}\right\},
$$

the inlet boundary $\Gamma_{i f}^{\varepsilon}(t)$

$$
\Gamma_{i f}^{\varepsilon}(t):=\left\{(x, y) \in \mathbb{R}^{2} \mid x=0,-\left(1-d^{\varepsilon}(0, t)\right) \leq y \leq\left(1-d^{\varepsilon}(0, t)\right)\right\},
$$

and the outflow boundary $\Gamma_{o f}^{\varepsilon}(t)$

$$
\Gamma_{o f}^{\varepsilon}(t):=\left\{(x, y) \in \mathbb{R}^{2} \mid x=1,-\left(1-d^{\varepsilon}(1, t)\right) \leq y \leq\left(1-d^{\varepsilon}(1, t)\right)\right\} .
$$

We use $\varepsilon$ as a superscript to emphasize the dependence of the respective variable on $\varepsilon$. Following (2.2), the dimensionless unit normal $\nu^{\varepsilon}$ becomes

$$
\nu^{\varepsilon}=\left(\varepsilon \partial_{x} d^{\varepsilon},-1\right)^{T} / \sqrt{1+\left(\varepsilon \partial_{x} d^{\varepsilon}\right)^{2}} .
$$

By $(2.3),(2.4)$, and $(2.5)$, the boundary condition on the lower part of $\Gamma_{g}^{\varepsilon}(t)$ is transformed into

$$
\varepsilon^{\alpha} D\left(-\varepsilon^{2} \partial_{x} d^{\varepsilon} \partial_{x} u^{\varepsilon}+\partial_{y} u^{\varepsilon}\right)=\varepsilon^{2} \partial_{t} d^{\varepsilon}\left(\rho-u^{\varepsilon}\right) \quad \text { on } \quad \Gamma_{g}^{\varepsilon}(t),
$$

where we have used the no-slip boundary condition for the velocity field at $\Gamma_{g}^{\varepsilon}(t)$. In this way, the dimensionless system of equations takes the form

$$
\begin{aligned}
& \partial_{t} u^{\varepsilon}-\varepsilon^{\alpha} D\left(\partial_{x x} u^{\varepsilon}+\frac{1}{\varepsilon^{2}} \partial_{y y} u^{\varepsilon}\right)+\partial_{x} q^{(1) \varepsilon} u^{\varepsilon}+\varepsilon^{-1} \partial_{y} q^{(2) \varepsilon} u^{\varepsilon}=0 \quad \text { in } \quad \Omega^{\varepsilon}(t), \\
& \partial_{x} q^{(1) \varepsilon}+\varepsilon^{-1} \partial_{y} q^{(2) \varepsilon}=0 \quad \text { in } \quad \Omega^{\varepsilon}(t), \\
& \varepsilon^{2} \mu \partial_{x x} q^{\varepsilon}+\mu \partial_{y y} q^{\varepsilon}=\left(\partial_{x} P^{\varepsilon}, \frac{1}{\varepsilon} \partial_{y} P^{\varepsilon}\right)^{T} \text { in } \Omega^{\varepsilon}(t), \\
& \partial_{t} d^{\varepsilon}=\sqrt{1+\left(\varepsilon \partial_{x} d^{\varepsilon}\right)^{2}} f\left(u^{\varepsilon}, \rho d^{\varepsilon}\right) \quad \text { on } \quad \Gamma_{g}^{\varepsilon}(t), \\
& \varepsilon^{\alpha} D\left(-\varepsilon^{2} \partial_{x} d^{\varepsilon} \partial_{x} u^{\varepsilon}+\partial_{y} u^{\varepsilon}\right)=\varepsilon^{2} \partial_{t} d^{\varepsilon}\left(\rho-u^{\varepsilon}\right) \quad \text { on } \quad \Gamma_{g}^{\varepsilon}(t) \text {, } \\
& q^{\varepsilon}=0 \quad \text { on } \quad \Gamma_{g}^{\varepsilon}(t), \\
& u^{\varepsilon}(x, y, 0)=u_{0} \quad \text { in } \quad \Omega^{\varepsilon}(0), \\
& d^{\varepsilon}(x, 0)=d_{0}(x) \text { in }(0, L) \text {. }
\end{aligned}
$$

Copyright $@$ by SIAM. Unauthorized reproduction of this article is prohibited. 
Remark 2.3. The well-posedness of the above model is in itself a nontrivial research subject. Nevertheless, this is beyond the scope of the present contribution. Here we note that the equations are based on physical laws such as the convectiondiffusion equation, the Stokes equation, and the conservation of mass. This is a natural approach for the modeling of the advection-diffusion-reactive flows. For smooth reaction rates, assuming that initially the free boundary is smooth, one may expect sufficient regularity of the solution, including the free boundary. In this case one can prove the existence and uniqueness of solution. For a one-dimensional situation, this has been proved in [26]. The delicate part in the rigorous analysis of this model is the regularity of free boundary and remains open for future considerations.

3. Upscaling. To obtain the upscaled equations, we use an asymptotic expansion for pressure, velocity, concentration, and the location of the free boundary. We define the effective quantities to describe the average behavior of the concentration and the free boundary variable. As will be seen below, the leading order terms of the expansion solve an upscaled equation of hyperbolic type. As the numerical experiments confirm, this hyperbolic model does not describe the average behavior in a satisfactory manner because of (Taylor) dispersion effects leading to an enhanced diffusion. To improve the hyperbolic model, we combine the leading order term and the first order term to define the effective quantities. Higher order terms are ignored in the expansion. The upscaled equation thereby obtained is of parabolic type and exhibits features of Taylor dispersion. The average of the flow profile satisfies a Darcytype equation with an explicit dependence of the permeability on the width of the strip.

3.1. The case $\mathrm{Pe}=\boldsymbol{O}\left(\varepsilon^{-1}\right)$. For the sake of presentation, the calculations below are performed for the case $\alpha=1$. The general situation, when $0<\alpha<2$ is analogous and leads to similar results, provided at the end of section 3.2.

We make the upscaling ansatz as follows:

$$
\begin{aligned}
P^{\varepsilon} & =P_{0}+\varepsilon P_{1}+O\left(\varepsilon^{2}\right), \\
q^{\varepsilon(1)} & =q_{0}^{(1)}+\varepsilon q_{1}^{(1)}+O\left(\varepsilon^{2}\right), \\
q^{\varepsilon(2)} & =q_{0}^{(2)}+\varepsilon q_{1}^{(2)}+O\left(\varepsilon^{2}\right), \\
d^{\varepsilon} & =d_{0}+\varepsilon d_{1}+O\left(\varepsilon^{2}\right), \\
u^{\varepsilon} & =u_{0}+\varepsilon u_{1}+O\left(\varepsilon^{2}\right) .
\end{aligned}
$$

We start with the flow problem. Substituting into the Stokes law (2.10), (2.11) the expansion for $P^{\varepsilon}, q^{\varepsilon(i)} i=1,2$, where $q^{\varepsilon(i)}=\left(q^{\varepsilon(1)}, q^{\varepsilon(2)}\right)^{T}$, we obtain

$$
\begin{aligned}
\partial_{x}\left(q_{0}^{(1)}+\varepsilon q_{1}^{(1)}\right)+\varepsilon^{-1} \partial_{y}\left(q_{0}^{(2)}+\varepsilon q_{1}^{(2)}\right) & =O\left(\varepsilon^{2}\right), \\
\varepsilon^{2} \mu \partial_{x x}\left(q_{0}^{(1)}+\varepsilon q_{1}^{(1)}\right)+\partial_{y y}\left(q_{0}^{(1)}+\varepsilon q_{1}^{(1)}\right) & =\partial_{x}\left(P_{0}+\varepsilon P_{1}\right)+O\left(\varepsilon^{2}\right), \\
\varepsilon^{2} \mu \partial_{x x}\left(q_{0}^{(2)}+\varepsilon q_{1}^{(2)}\right)+\partial_{y y}\left(q_{0}^{(2)}+\varepsilon q_{1}^{(2)}\right) & =\varepsilon^{-1} \partial_{y}\left(P_{0}+\varepsilon P_{1}\right)+O\left(\varepsilon^{2}\right) .
\end{aligned}
$$

We proceed by equating terms of similar order in the equations above. For the $\varepsilon^{-1}$ order term in $\Omega^{\varepsilon}(t)$, we get the $\varepsilon^{-1}$ terms

$$
\begin{aligned}
\partial_{y} q_{0}^{(2)} & =0, \\
\partial_{y} P_{0} & =0 .
\end{aligned}
$$

Copyright $@$ by SIAM. Unauthorized reproduction of this article is prohibited. 
At $y=d^{\varepsilon}-1$,

$$
q^{\varepsilon}=0
$$

and together with $(3.2)$

$$
q_{0}^{(2)} \equiv 0
$$

Also (3.3) implies

$$
P_{0}=P_{0}(x, t) .
$$

Further, in $\Omega^{\varepsilon}(t)$ we have the $\varepsilon^{0}$ terms

$$
\begin{aligned}
\partial_{x} q_{0}^{(1)}+\partial_{y} q_{1}^{(2)} & =0, \\
\mu \partial_{y y} q_{0}^{(2)} & =\partial_{y} P_{1}, \\
\mu \partial_{y y} q_{0}^{(1)} & =\partial_{x} P_{0}
\end{aligned}
$$

and the $\varepsilon^{1}$ terms

$$
\mu \partial_{y y} q_{1}^{(1)}=\partial_{x} P_{1} .
$$

Using (3.4) and (3.7), we conclude $P_{1}=P_{1}(x, t)$. Combining (3.8) and (3.9), we obtain

$$
\mu \partial_{y y}\left(q_{0}^{(1)}+\varepsilon q_{1}^{(1)}\right)=\partial_{x}\left(P_{0}+\varepsilon P_{1}\right) .
$$

However, the boundary conditions need a careful treatment. To take into account the terms up to first order, we start with defining the effective quantity describing the free boundary variable $d_{e}$ with

$$
d_{e}=d_{0}+\varepsilon d_{1} .
$$

Note that with this definition, $d^{\varepsilon}=d_{e}+O\left(\varepsilon^{2}\right)$. Other effective quantities are defined below (see (3.14)).

Since we are primarily interested in effects that are up to first order in $\varepsilon$, we define the boundary conditions at $y=d_{e}-1$. Note that rewriting the boundary conditions at $d_{e}-1$ introduces an error of the order $O\left(\varepsilon^{2}\right)$. Using the ansatz expansion (3.1) and the Taylor expansion around $d_{e}-1$ provides

$$
\begin{aligned}
0=q^{\varepsilon}\left(x, d^{\varepsilon}-1, t\right) & =q_{0}\left(x, d^{\varepsilon}-1, t\right)+\varepsilon q_{1}\left(x, d^{\varepsilon}-1, t\right)+O\left(\varepsilon^{2}\right) \\
& =q_{0}\left(x, d_{0}+\varepsilon d_{1}-1, t\right)+\varepsilon q_{1}\left(x, d_{0}+\varepsilon d_{1}-1, t\right)+O\left(\varepsilon^{2}\right) \\
& =q_{0}\left(x, d_{e}-1, t\right)+\varepsilon q_{1}\left(x, d_{e}-1, t\right)+O\left(\varepsilon^{2}\right) .
\end{aligned}
$$

Using the boundary condition (3.12) at $d_{e}-1$ and the symmetry condition at $y=0$, (3.10) gives

$$
q_{0}^{(1)}+\varepsilon q_{1}^{(1)}=\left(y^{2}-\left(1-d_{e}\right)^{2}\right) \frac{\partial_{x}\left(P_{0}+\varepsilon P_{1}\right)}{2 \mu}+O\left(\varepsilon^{2}\right) .
$$

The $O\left(\varepsilon^{2}\right)$ terms are due to the approximation of the boundary condition (3.12).

Copyright (c) by SIAM. Unauthorized reproduction of this article is prohibited. 
Similar to $d_{e}$, we define the effective quantities

$$
\begin{aligned}
u_{e}(x, t) & :=u_{0}+\varepsilon \bar{u}_{1}, \\
\bar{q}_{e}(x, t) & :=\int_{d_{e}-1}^{0} q_{e}^{(1)}(x, y, t) d y, \\
P_{e}(x, t) & :=P_{0}+\varepsilon P_{1},
\end{aligned}
$$

where

$$
q_{e}:=q_{0}+\varepsilon q_{1} \quad \text { and } \quad \bar{u}_{1}:=\frac{1}{1-d_{e}} \int_{d_{e}-1}^{0} u_{1}(x, y, t) d y .
$$

The definition of $u_{e}$ assumes that $u_{0}=u_{0}(x, t)$, which will be justified below. Moreover, as will be shown below, $q_{e}^{(2)}$ does not play any role in the upscaled equations; therefore we define $\bar{q}_{e}$ only in terms of $q_{e}^{(1)}$. Neglecting higher order terms, (3.13) and (3.14) give

$$
q_{e}^{(1)}=\left(y^{2}-\left(1-d_{e}\right)^{2}\right) \frac{\partial_{x} P_{e}}{2 \mu} .
$$

Integrating $q_{e}^{(1)}$ from the above expression for $y$ over $\left(d_{e}-1,0\right)$ provides

$$
\bar{q}_{e}:=\int_{d_{e}-1}^{0} q_{e}^{(1)} d y=-\left(1-d_{e}\right)^{3} \frac{\partial_{x} P_{e}}{3 \mu},
$$

which may be interpreted as the Darcy law for the thin strip case with variable geometry. Note that the permeability reported in literature [3] is proportional to $\left(1-d_{e}\right)^{2}$, but since we integrate the velocity over the thickness of the strip, here we get it proportional to $\left(1-d_{e}\right)^{3}$.

We consider the convection diffusion equation, first average it along the transverse direction, and substitute the expansion for variables. Averaging (2.9) along the transverse direction gives

$$
\int_{d^{\varepsilon}-1}^{0} \partial_{t} u^{\varepsilon}-\varepsilon D\left(\partial_{x x} u^{\varepsilon}+\frac{1}{\varepsilon^{2}} \partial_{y y} u^{\varepsilon}\right)+\partial_{x}\left(q^{(1) \varepsilon} u^{\varepsilon}\right)+\partial_{y}\left(q^{(2) \varepsilon} u^{\varepsilon}\right)=0 .
$$

Exchanging the derivative and the integral in the first two terms gives

$$
\begin{aligned}
\partial_{t} \int_{d^{\varepsilon}-1}^{0} u^{\varepsilon} d y+\left.\partial_{t} d^{\varepsilon} u^{\varepsilon}\right|_{\left(y=d^{\varepsilon}-1\right)} & -\varepsilon D \partial_{x} \int_{d^{\varepsilon}-1}^{0} \partial_{x} u^{\varepsilon} d y \\
& -\left.\left(\varepsilon D \partial_{x} d^{\varepsilon} \partial_{x} u^{\varepsilon}+\frac{D}{\varepsilon} \partial_{y} u^{\varepsilon}\right)\right|_{\left(y=d^{\varepsilon}-1\right)} \\
& +\partial_{x} \int_{d^{\varepsilon}-1}^{0} q^{(1) \varepsilon} u^{\varepsilon} d y \\
& +\left.\left(\partial_{x} d^{\varepsilon} q^{(1) \varepsilon} u^{\varepsilon}\right)\right|_{\left(y=d^{\varepsilon}-1\right)}+\left.q^{(2) \varepsilon} u^{\varepsilon}\right|_{\left(y=d^{\varepsilon}-1\right)} ^{0}=0 .
\end{aligned}
$$

To simplify the expression above, we use the boundary condition (2.13) for the third term. The last two terms vanish; the next to last term does not contribute due to 
the no-slip boundary condition, whereas the last term is 0 due to both the symmetry condition at $y=0$ and the no-slip boundary condition at $y=d^{\varepsilon}-1$. This leads to

$$
\partial_{t} \int_{d^{\varepsilon}-1}^{0} u^{\varepsilon} d y+\partial_{t} d^{\varepsilon} \rho-\varepsilon D \partial_{x} \int_{d^{\varepsilon}-1}^{0} \partial_{x} u^{\varepsilon} d y+\partial_{x} \int_{d^{\varepsilon}-1}^{0} q^{(1) \varepsilon} u^{\varepsilon} d y=0 .
$$

Substituting the ansatz (3.1) in (3.18) and retaining the terms up to $O(\varepsilon)$, we obtain

$$
\begin{aligned}
\partial_{t} \int_{d_{e}-1}^{0}\left(u_{0}+\varepsilon u_{1}\right) d y+\partial_{t}\left(d_{0}+\varepsilon d_{1}\right) \rho & -\varepsilon D \partial_{x} \int_{d_{e}-1}^{0}\left(\partial_{x} u_{0}+\varepsilon \partial_{x} u_{1}\right) d y \\
& +\partial_{x} \int_{d_{e}-1}^{0} q_{0}^{(1)} u_{0}+\varepsilon\left(q_{0}^{(1)} u_{1}+q_{1}^{(1)} u_{0}\right) d y=O\left(\varepsilon^{2}\right) .
\end{aligned}
$$

Observe that by retaining only two terms of the expansion, in each term of the expression (3.18), the remainder introduces an error of the order $O\left(\varepsilon^{2}\right)$. Also changing the domain of integration from $d^{\varepsilon}-1$ to $d_{e}-1$ introduces an error of the order $O\left(\varepsilon^{2}\right)$. Adding the $\varepsilon^{2} q_{1}^{(1)} u_{1}$ term in (3.19), we obtain up to $O\left(\varepsilon^{2}\right)$

$\partial_{x} \int_{d_{e}-1}^{0}\left(q_{0}^{(1)}+\varepsilon q_{1}^{(1)}\right) u_{0}+\varepsilon\left(q_{0}^{(1)}+\varepsilon q_{1}^{(1)}\right) u_{1} d y=\partial_{x} \int_{d_{e}-1}^{0}\left(q_{e}^{(1)} u_{0}+\varepsilon q_{e} u_{1}\right) d y$

$$
=\partial_{x}\left\{u_{e} \bar{q}_{e}-\varepsilon \bar{u}_{1} \bar{q}_{e}+\varepsilon \int_{d_{e}-1}^{0} q_{e} u_{1} d y\right\} .
$$

The above equation contains the unknown term $u_{1}$. For computing it, the leading order term $u_{0}$ is needed. The $\varepsilon^{-1}$ and $\varepsilon^{0}$ terms for the convection diffusion equation using the ansatz expansion (3.1) provide

$$
\begin{array}{r}
\partial_{y y} u_{0}=\partial_{y} q_{0}^{(2)} u_{0}, \\
\partial_{t} u_{0}-D \partial_{y y} u_{1}+\partial_{x}\left(q_{0}^{(1)} u_{0}\right)+\partial_{y}\left(q_{1}^{(2)} u_{0}\right)=0,
\end{array}
$$

and using (3.21) together with symmetry condition at $y=0$, we conclude $u_{0} \equiv$ $u_{0}(x, t)$. Simplifying (3.22) using (3.6), we obtain

$$
\partial_{t} u_{0}-D \partial_{y y} u_{1}+q_{0}^{(1)} \partial_{x} u_{0}=0 .
$$

As for the flow, we now treat the boundary condition for the convection diffusion equation. Using (2.13) and a Taylor expansion around $y=d_{e}-1$, we have

$$
\begin{aligned}
0 & =\left.\left\{D\left(-\varepsilon^{2} \partial_{x} d^{\varepsilon} \partial_{x} u^{\varepsilon}+\partial_{y} u^{\varepsilon}\right)-\varepsilon \partial_{t} d^{\varepsilon}\left(\rho-u^{\varepsilon}\right)\right\}\right|_{\left(x, d^{\varepsilon}-1, t\right)} \\
& =\left.\left\{D\left(-\varepsilon^{2} \partial_{x} d^{\varepsilon} \partial_{x} u^{\varepsilon}+\partial_{y} u^{\varepsilon}\right)-\varepsilon \partial_{t} d^{\varepsilon}\left(\rho-u^{\varepsilon}\right)\right\}\right|_{\left(x, d_{e}-1, t\right)}+O\left(\varepsilon^{2}\right) \\
& =\left.\left\{D\left(-\varepsilon^{2} \partial_{x} d_{0} \partial_{x} u_{0}+\partial_{y} u_{0}+\varepsilon \partial_{y} u_{1}\right)-\varepsilon \partial_{t} d_{0}\left(\rho-u_{0}\right)\right\}\right|_{\left(x, d_{e}-1, t\right)}+O\left(\varepsilon^{2}\right) .
\end{aligned}
$$

Since $u_{0}=u_{0}(x, t)$, we conclude

$$
D \partial_{y} u_{1}-\left.\partial_{t} d_{0}\left(\rho-u_{0}\right)\right|_{y=d_{e}-1}=O(\varepsilon) .
$$

Integrate (3.23) over y from $d_{e}-1$ to 0 and use the symmetry condition at $y=0$ and boundary condition at $y=d_{e}-1$ to get the compatibility condition

$$
\left(1-d_{e}\right) \partial_{t} u_{0}+\partial_{t} d_{0}\left(\rho-u_{0}\right)+\int_{d_{e}-1}^{0} q_{0}^{(1)} \partial_{x} u_{0} d y=O(\varepsilon)
$$

Copyright $@$ by SIAM. Unauthorized reproduction of this article is prohibited. 
The above equation provides the leading order solution for the convection-diffusion equation. However, as remarked earlier, to improve the upscaled model we also include the first order term. To do so, we multiply (3.23) by $1-d_{e}$, subtract from (3.25), and then integrate twice, first from 0 to $y$ and then from $d_{e}-1$ to $y$ using the symmetry conditions at $y=0$,

$\partial_{t} d_{0} \frac{\left(\rho-u_{0}\right) y^{2}}{2}+\left(1-d_{e}\right) D u_{1}+\left(\frac{\left(1-d_{e}\right)^{3} y^{2}}{6}-\frac{\left(1-d_{e}\right) y^{4}}{12}\right) \frac{\partial_{x} P_{0}}{2 \mu} \partial_{x} u_{0}+C(x, t)=O(\varepsilon)$,

where $C(x, t)$ is a constant of integration. Disregarding $O(\varepsilon)$ terms, $u_{1}$ is given by

$$
u_{1}=\frac{-C}{D\left(1-d_{e}\right)}+\frac{\Xi(y)}{D\left(1-d_{e}\right)}+O(\varepsilon)
$$

where $\Xi(y)$ is defined as

$$
\Xi(y)=-\partial_{t} d_{0}\left(\rho-u_{0}\right) \frac{y^{2}}{2}-\left\{\frac{\left(1-d_{e}\right)^{3} y^{2}}{6}-\frac{\left(1-d_{e}\right) y^{4}}{12}\right\} \frac{\partial_{x} P_{0} \partial_{x} u_{0}}{2 \mu}
$$

Straightforwardly

$$
\int_{d_{e}-1}^{0} \Xi(y) d y=\frac{\left(1-d_{e}\right)^{3}}{180}\left\{-30 \partial_{t} d_{0}\left(\rho-u_{0}\right)-7 \frac{\left(1-d_{e}\right)^{3}}{2 \mu} \partial_{x} P_{0} \partial_{x} u_{0}\right\}
$$

and hence

$$
\bar{u}_{1}=\frac{1}{1-d_{e}}\left\{\frac{-C}{D}+\frac{\left(1-d_{e}\right)^{2}}{180 D}\left\{-30 \partial_{t} d_{0}\left(\rho-u_{0}\right)-7\left(1-d_{e}\right)^{3} \frac{\partial_{x} P_{0} \partial_{x} u_{0}}{2 \mu}\right\}\right\}+O(\varepsilon),
$$

where $\bar{u}_{1}$ is defined in (3.14). The last term in (3.20) becomes

$$
\int_{d_{e}-1}^{0} q_{e}^{(1)} u_{1} d y=\int_{d_{e}-1}^{0}\left(y^{2}-\left(1-d_{e}\right)^{2}\right) \frac{\partial_{x} P_{e}}{2 \mu}\left\{\frac{-C}{D\left(1-d_{e}\right)}+\frac{\Xi(y)}{D\left(1-d_{e}\right)}\right\} d y+O(\varepsilon) .
$$

Clearly

$$
\int_{d_{e}-1}^{0}\left(y^{2}-\left(1-d_{e}\right)^{2}\right) \Xi(y) d y=\frac{\left(1-d_{e}\right)^{5}}{630}\left\{42 \partial_{t} d_{0}\left(\rho-u_{0}\right)+11\left(1-d_{e}\right)^{3} \frac{\partial_{x} P_{0}}{2 \mu} \partial_{x} u_{0}\right\} .
$$


Using (3.28), (3.30), (3.31), and (3.20) gives

$$
\begin{aligned}
& \partial_{x}\left\{u_{e} \bar{q}_{e}-\varepsilon \bar{u}_{1} \bar{q}_{e}+\varepsilon \int_{d_{e}-1}^{0} q_{e}^{(1)} u_{1} d y\right\} \\
&=\partial_{x}\left\{u_{e} \bar{q}_{e}-\frac{\varepsilon}{1-d_{e}}\left\{\frac { - \partial _ { x } P _ { e } } { 3 \mu } ( 1 - d _ { e } ) ^ { 3 } \left\{\frac{-C}{D}+\frac{\left(1-d_{e}\right)^{2}}{180 D}\right.\right.\right. \\
&\left.\left.\left.\times\left\{-30 \partial_{t} d_{0}\left(\rho-u_{0}\right)-7\left(1-d_{e}\right)^{3} \partial_{x} u_{0} \frac{\partial_{x} P_{0}}{2 \mu}\right\}\right\}\right\}\right\} \\
&+ \varepsilon \partial_{x}\left\{\frac{C}{D} \frac{\partial_{x} P_{e}}{3 \mu}\left(1-d_{e}\right)^{2}+\frac{\left(1-d_{e}\right)^{4}}{630 D} \frac{\partial_{x} P_{e}}{2 \mu}\right. \\
&\left.\times\left\{42 \partial_{t} d_{0}\left(\rho-u_{0}\right)+11\left(1-d_{e}\right)^{3} \partial_{x} u_{0} \frac{\partial_{x} P_{0}}{2 \mu}\right\}\right\}+O(\varepsilon) \\
&=\partial_{x}\left\{u_{e} \bar{q}_{e}+\varepsilon \frac{\partial_{x} P_{e}}{D \mu}\left\{-\frac{1}{45}\left(1-d_{e}\right)^{4} \partial_{t} d_{0}\left(\rho-u_{0}\right)\right.\right. \\
&\left.\left.+\left(-\frac{4}{945}\right)\left(1-d_{e}\right)^{7} \frac{\partial_{x} P_{e}}{2 \mu} \partial_{x} u_{0}\right\}\right\}+O(\varepsilon) .
\end{aligned}
$$

Hence the averaged equation reads

$$
\begin{aligned}
\partial_{t} \int_{d_{e}-1}^{0}\left(u_{0}+\varepsilon u_{1}\right) d y+ & \partial_{t}\left(d_{0}+\varepsilon d_{1}\right) \rho-\varepsilon D \partial_{x} \int_{d_{e}-1}^{0}\left(\partial_{x} u_{0}+\varepsilon \partial_{x} u_{1}\right) u_{1} d y \\
& +\partial_{x} \int_{d_{e}-1}^{0} q_{0}^{(1)} u_{0}+\varepsilon\left(q_{0}^{(1)} u_{1}+q_{1}^{(1)} u_{0}\right) d y=O\left(\varepsilon^{2}\right) .
\end{aligned}
$$

Recalling (3.14), this translates into

$$
\begin{aligned}
& \partial_{t}\left(\left(1-d_{e}\right) u_{e}\right)+\partial_{t}\left(d_{e} \rho\right)-\varepsilon D \partial_{x}\left(\left(1-d_{e}\right) \partial_{x} u_{e}\right) \\
& \quad+\partial_{x}\left\{u_{e} \bar{q}_{e}+\varepsilon \frac{\partial_{x} P_{e}}{D \mu}\left\{-\frac{1}{45} \partial_{t} d_{0}\left(\rho-u_{0}\right)\left(1-d_{e}\right)^{4}-\frac{4}{945}\left(1-d_{e}\right)^{7} \partial_{x} u_{0} \frac{\partial_{x} P_{e}}{2 \mu}\right\}\right\}=O\left(\varepsilon^{2}\right) .
\end{aligned}
$$

For $d_{e}$, by using the Taylor expansion of $f\left(u^{\varepsilon}, d^{\varepsilon}\right)$ around $\left(u_{e}, d_{e}\right)$, we obtain formally

$$
\begin{aligned}
\partial_{t} \rho d_{e}=f\left(u^{\varepsilon}, \rho d^{\varepsilon}\right)= & f\left(u_{0}+\varepsilon \bar{u}_{1}, \rho\left(d_{0}+\varepsilon d_{1}\right)\right) \\
& +\varepsilon\left(\left.u_{1}\right|_{y=d_{e}-1}-\bar{u}_{1}\right) \partial_{1} f\left(u_{0}+\varepsilon \bar{u}_{1}, \rho\left(d_{0}+\varepsilon d_{1}\right)\right)+O\left(\varepsilon^{2}\right) \\
= & f\left(u_{e}, \rho d_{e}\right)+\varepsilon\left(\left.u_{1}\right|_{y=d_{e}-1}-\bar{u}_{1}\right) \partial_{1} f\left(u_{e}, \rho d_{e}\right)+O\left(\varepsilon^{2}\right) .
\end{aligned}
$$

By (3.26) and (3.29) we have

$$
\left.u_{1}\right|_{y=d_{e}-1}-\bar{u}_{1}=\left(1-d_{e}\right)\left\{-\frac{1}{3 D}\left(\rho-u_{e}\right) \partial_{t} d_{e}+\frac{1}{15 D} \varepsilon \bar{q}_{e} \partial_{x} u_{e}\right\}+O(\varepsilon),
$$

where we have used the Darcy law (3.15). Hence the upscaled equation for $\partial_{t} d_{e}$ becomes

$$
\partial_{t}\left(\rho d_{e}\right)=f\left(u_{e}, \rho d_{e}\right)+\varepsilon\left(1-d_{e}\right)\left\{-\frac{1}{3 D}\left(\rho-u_{e}\right) \partial_{t} d_{e}+\frac{1}{15 D} \bar{q}_{e} \partial_{x} u_{e}\right\} \partial_{1} f\left(u_{e}, \rho d_{e}\right)+O\left(\varepsilon^{2}\right) .
$$


For convenience, we write at one place the upscaled equations (3.15), (3.32), and $(3.35)$,

$$
\begin{aligned}
\bar{q}_{e}= & -\left(1-d_{e}\right)^{3} \frac{\partial_{x} P_{e}}{3 \mu}+O\left(\varepsilon^{2}\right), \\
\partial_{t}\left(\left(1-d_{e}\right) u_{e}+d_{e} \rho\right)= & \partial_{x}\left\{-u_{e} \bar{q}_{e}+\varepsilon\left(1-d_{e}\right) D\left(1+\frac{2 \bar{q}_{e}^{2}}{105 D^{2}}\right) \partial_{x} u_{e}\right. \\
& \left.-\varepsilon \frac{\bar{q}_{e}}{15 D} \partial_{t} d_{e}\left(\rho-u_{e}\right)\left(1-d_{e}\right)\right\}+O\left(\varepsilon^{2}\right), \\
\partial_{t}\left(\rho d_{e}\right)= & f\left(u_{e}, \rho d_{e}\right)+\varepsilon\left(1-d_{e}\right)\left\{-\frac{1}{3 D}\left(\rho-u_{e}\right) \partial_{t} d_{e}+\frac{1}{15 D} \bar{q}_{e} \partial_{x} u_{e}\right\} \\
& \times \partial_{1} f\left(u_{e}, \rho d_{e}\right)+O\left(\varepsilon^{2}\right) .
\end{aligned}
$$

To eliminate the pressure from the first equation, we take a small slice of the half-strip of length $\delta x$, denoted by

$$
Y=\left\{(v, w)\left|x_{1} \leq v \leq x_{1}+\delta x,\right| w \mid \leq\left(1-d_{e}\right)\right\} .
$$

The continuity equation and the divergence theorem give

$$
0=\int_{Y} \nabla \cdot q^{\varepsilon}=\left.\int_{-\left(1-d_{e}\right)}^{1-d_{e}} q_{e}^{(1)}\right|_{x=x_{1}+\delta x} d y-\left.\int_{-\left(1-d_{e}\right)}^{1-d_{e}} q_{e}^{(1)}\right|_{x=x_{1}} d y+O\left(\varepsilon^{2}\right),
$$

where we have used the no-slip boundary condition to make the boundary terms on the lateral surface equal to 0 . Dividing by $\delta x$ and then taking the limit $\delta x \rightarrow 0$, we obtain

$$
\partial_{x} \bar{q}_{e}=O\left(\varepsilon^{2}\right)
$$

Hence the upscaled system of equations after neglecting the $O\left(\varepsilon^{2}\right)$ terms from the effective equations for $\bar{q}_{e}, u_{e}, d_{e}$ becomes

$$
\begin{aligned}
\partial_{x} \bar{q}_{e}= & 0, \\
\partial_{t}\left(\left(1-d_{e}\right) u_{e}+d_{e} \rho\right)= & \partial_{x}\left\{-u_{e} \bar{q}_{e}+\varepsilon\left(1-d_{e}\right) D\left(1+\frac{2 \bar{q}_{e}^{2}}{105 D^{2}}\right) \partial_{x} u_{e}\right. \\
& \left.-\varepsilon \frac{\bar{q}_{e}}{15 D} \partial_{t} d_{e}\left(\rho-u_{e}\right)\left(1-d_{e}\right)\right\} \\
\partial_{t}\left(\rho d_{e}\right)= & f\left(u_{e}, \rho d_{e}\right)+\varepsilon\left(1-d_{e}\right)\left\{-\frac{1}{3 D}\left(\rho-u_{e}\right) \partial_{t} d_{e}+\frac{1}{15 D} \bar{q}_{e} \partial_{x} u_{e}\right\} \\
& \times \partial_{1} f\left(u_{e}, \rho d_{e}\right) .
\end{aligned}
$$

Remark 3.1. To compare the upscaled model for the variable geometry with that in the fixed geometry case, we refer to [10], where the following system is derived (also see Appendix A for an alternative approach):

$$
\begin{aligned}
\partial_{t}\left(u_{e}+v_{e}\right) & =\partial_{x}\left\{-u_{e} \bar{q}_{e}+\varepsilon D\left(1+\frac{2 \bar{q}_{e}^{2}}{105 D^{2}}\right) \partial_{x} u_{e}-\varepsilon \frac{1}{15} \frac{\bar{q}_{e}}{D} f\left(u_{e}, v_{e}\right)\right\} \\
\left(1+\varepsilon \frac{1}{3 D} \partial_{1} f\left(u_{e}, v_{e}\right)\right) \partial_{t} v_{e} & =f\left(u_{e}, v_{e}\right)+\varepsilon \frac{\bar{q}_{e}}{15 D} \partial_{x} u_{e} \partial_{1} f\left(u_{e}, v_{e}\right) .
\end{aligned}
$$

Following the ideas in [25], where in the case $\mathbf{P e}=\mathbf{O}(\mathbf{1})$ the fixed geometry case is obtained as the limit of a variable geometry model, we assume that $d_{e} \searrow 0(\rho \rightarrow \infty)$, whereas $\rho d_{e} \rightarrow v_{e}$. Then the upscaled equations for the variable geometry case reduce to that of the fixed geometry case.

Copyright (c) by SIAM. Unauthorized reproduction of this article is prohibited. 
3.2. The case $\mathrm{Pe}=\boldsymbol{O}\left(\varepsilon^{-\alpha}\right)$. In section 3.1 , we have considered only the case $\alpha=1$. This choice was made strictly for the ease of presentation. However, other scalings may be required for different applications. For example, bringing the experiments carried out by Taylor [32] to a dimensionless model leads to either $\alpha=1.6$ or $\alpha=1.9$ (see [10]). Nevertheless, the asymptotic expansion procedure in the previous section can be extended to the case $\alpha \in(0,2)$ but with a slightly different expansion:

$$
\begin{aligned}
P^{\varepsilon} & =P_{0}+\varepsilon P_{1}+O\left(\varepsilon^{2}\right), \\
q^{\varepsilon(1)} & =q_{0}^{(1)}+\varepsilon q_{1}^{(1)}+O\left(\varepsilon^{2}\right), \\
q^{\varepsilon(2)} & =q_{0}^{(2)}+\varepsilon q_{1}^{(2)}+O\left(\varepsilon^{2}\right), \\
d^{\varepsilon} & =d_{0}+\varepsilon^{2-\alpha} d_{1}+O\left(\varepsilon^{2(2-\alpha)}\right), \\
u^{\varepsilon} & =u_{0}+\varepsilon^{2-\alpha} u_{1}+O\left(\varepsilon^{2(2-\alpha)}\right) .
\end{aligned}
$$

For $\alpha \geq 2$ we enter into the turbulent mixing regime, and the approach considered in this paper fails.

Following the steps outlined in section 3.1, analogous to (3.19) we obtain

$$
\begin{aligned}
\partial_{t} \int_{d^{\varepsilon}-1}^{0}\left(u_{0}+\varepsilon^{2-\alpha} u_{1}\right) d y & +\partial_{t}\left(d_{0}+\varepsilon^{2-\alpha} d_{1}\right) \rho-\varepsilon^{\alpha} D \partial_{x} \int_{d^{\varepsilon}-1}^{0}\left(\partial_{x} u_{0}+\varepsilon^{2-\alpha} \partial_{x} u_{1}\right) d y \\
& +\partial_{x} \int_{d^{\varepsilon}-1}^{0} q_{0}^{(1)} u_{0}+\left(\varepsilon^{2-\alpha} q_{0}^{(1)} u_{1}+\varepsilon q_{1}^{(1)} u_{0}\right) d y=+O\left(\varepsilon^{3-\alpha}\right),
\end{aligned}
$$

and the last term in the above can be expressed similarly to (3.20):

$$
\begin{aligned}
\partial_{x} \int_{d_{e}-1}^{0}\left(q_{0}^{(1)}+\varepsilon q_{1}^{(1)}\right) u_{0} & +\varepsilon^{2-\alpha}\left(q_{0}^{(1)}+\varepsilon q_{1}^{(1)}\right) u_{1} d y \\
& =\partial_{x}\left\{u_{e} \bar{q}_{e}-\varepsilon^{2-\alpha} \bar{u}_{1} \bar{q}_{e}+\varepsilon^{2-\alpha} \int_{d_{e}-1}^{0} q_{e}^{(1)} u_{1} d y\right\} .
\end{aligned}
$$

Defining the effective quantities as

$$
\begin{aligned}
& d_{e}:=d_{0}+\varepsilon^{2-\alpha} d_{1}, \\
& u_{e}:=u_{0}+\varepsilon^{2-\alpha}\left\langle u_{1}\right\rangle, \\
& \bar{q}_{e}=\int_{d_{e}-1}^{0} q_{e}^{(1)}(x, y, t) d y, \\
& P_{e}:=P_{0}+\varepsilon P_{1},
\end{aligned}
$$

where

$$
q_{e}:=q_{0}+\varepsilon q_{1} \quad \text { and } \quad\left\langle u_{1}\right\rangle=\frac{1}{1-d_{e}} \int_{d_{e}-1}^{0} u_{1}(x, y, t) d y,
$$

the upscaled equations are

$$
\begin{aligned}
\partial_{x} \bar{q}_{e} & =0, \\
\bar{q}_{e} & =-\frac{\left(1-d_{e}\right)^{3}}{3 \mu} \partial_{x} P_{e}
\end{aligned}
$$

Copyright $\odot$ by SIAM. Unauthorized reproduction of this article is prohibited. 


$$
\begin{aligned}
\partial_{t}\left(\left(1-d_{e}\right) u_{e}+d_{e} \rho\right)= & \partial_{x}\left\{-u_{e} \bar{q}_{e}\right\} \\
& +\partial_{x}\left\{\varepsilon^{\alpha}\left(1-d_{e}\right) D\left(1+\varepsilon^{2(1-\alpha)} \frac{2 \bar{q}_{e}^{2}}{105 D^{2}}\right) \partial_{x} u_{e}\right\} \\
& +\partial_{x}\left\{-\varepsilon^{2-\alpha} \frac{\bar{q}_{e}}{15 D} \partial_{t} d_{e}\left(\rho-u_{e}\right)\left(1-d_{e}\right)\right\} \\
\partial_{t}\left(\rho d_{e}\right)= & f\left(u_{e}, \rho d_{e}\right) \\
& +\varepsilon^{2-\alpha}\left(1-d_{e}\right)\left\{-\frac{1}{3 D}\left(\rho-u_{e}\right) \partial_{t} d_{e}+\frac{1}{15 D} \bar{q}_{e} \partial_{x} u_{e}\right\} \partial_{1} f\left(u_{e}, \rho d_{e}\right) .
\end{aligned}
$$

It is again to be observed that the Darcy equation is retrieved by upscaling of the flow field, and for the simple geometry of the strip, we obtain an explicit characteristic dependence of the permeability on the geometry, namely, $\frac{\left(1-d_{e}\right)^{3}}{3}$. The traveling wave solution approach for the upscaled equations in the case of moderate Peclét number has been studied in [25]. For the fixed geometry situation, a detailed analysis has been carried out in [9]. The analysis for the present, variable geometry model is a subject of future consideration.

4. Numerical validation. For the numerical experiments, we consider the case of crystal precipitation and dissolution. For this case, the reaction rate function $f(u, v)$ is given by

$$
f(u, v)=k\left(r_{p}-r_{d}\right),
$$

where $r_{p}$ and $r_{d}$ denote, respectively, the precipitation and dissolution rates. Here we take $r_{p}=r(u)=u$, whereas for $r_{d}$ we follow [9] and choose $r_{d}=\frac{1}{2} \tilde{H}(v)$ with $\tilde{H}(\cdot)$ denoting the Heaviside graph. Since the reaction rate in this form is multivalued, we use a regularized form $H(\cdot)$ defined as

$$
H(v)=\left\{\begin{array}{cc}
0, & v \leq 0 \\
\frac{v}{\delta}, & 0<v<\delta, \\
1, & v \geq \delta,
\end{array}\right.
$$

where, for instance, $\delta=o(\varepsilon)$.

For comparison purposes, different macroscopic equations can be considered.

4.1. Hyperbolic model. The first one is obtained by asymptotic expansion as carried out in the previous section but considering only the leading order term. This involves a hyperbolic equation for the solute,

(Hyperbolic)

$$
\begin{aligned}
\partial_{x} \bar{q}_{e} & =0 \\
\partial_{t}\left(\left(1-d_{e}\right) u_{e}+d_{e} \rho\right) & =\partial_{x}\left\{-\bar{q}_{e} u_{e}\right\} \\
\partial_{t}\left(\rho d_{e}\right) & =k\left(r\left(u_{e}\right)-H\left(\rho d_{e}\right)\right) .
\end{aligned}
$$

We will refer to this model as Hyperbolic for the numerical computations.

4.2. Simple averaging. Next a straightforward upscaling by transverse averaging provides an effective model that does not include the dispersion term but only the original parabolic terms. Furthermore, for the chemical reactions, we obtain a straightforward model. This effective model will be named in what follows as Simple Averaging. 
(Simple Averaging) $\quad \partial_{x} \bar{q}_{e}=0$,

$$
\begin{aligned}
\partial_{t}\left(\left(1-d_{e}\right) u_{e}+d_{e} \rho\right) & =\partial_{x}\left\{-u_{e} \bar{q}_{e}+\varepsilon\left(1-d_{e}\right) D \partial_{x} u_{e}\right\}, \\
\partial_{t}\left(\rho d_{e}\right) & =k\left(r\left(u_{e}\right)-H\left(\rho d_{e}\right)\right) .
\end{aligned}
$$

4.3. Upscaled model. Finally we use the upscaled model derived for the variable geometry case:

\section{(Upscaled)}

$$
\begin{aligned}
\partial_{x} \bar{q}_{e}= & 0, \\
\partial_{t}\left(\left(1-d_{e}\right) u_{e}+d_{e} \rho\right)= & \partial_{x}\left\{-u_{e} \bar{q}_{e}+\varepsilon\left(1-d_{e}\right) D\left(1+\frac{2 \bar{q}_{e}^{2}}{105 D^{2}}\right) \partial_{x} u_{e}\right. \\
& \left.-\varepsilon \frac{\bar{q}_{e}}{15 D} \partial_{t} d_{e}\left(\rho-u_{e}\right)\left(1-d_{e}\right)\right\}, \\
\partial_{t}\left(\rho d_{e}\right)= & k\left(r\left(u_{e}\right)-H\left(\rho d_{e}\right)\right)+\varepsilon\left(1-d_{e}\right)\left\{-\frac{1}{3 D}\left(\rho-u_{e}\right) \partial_{t} d_{e}+\frac{1}{15 D} \bar{q}_{e} \partial_{x} u_{e}\right\} k .
\end{aligned}
$$

Henceforth, for the numerical computations, we name it as Upscaled.

4.4. 2-D average. To compare the upscaled equations with the average of the solution of microscopic equations, we first compute the full solution of the system of (2.9)-(2.14) with the given initial data. To obtain the 2-D average, we compute

$$
\bar{u}=\frac{1}{2\left(1-d^{\varepsilon}\right)} \int_{-\left(1-d^{\varepsilon}\right)}^{\left(1-d^{\varepsilon}\right)} u^{\varepsilon} d y .
$$

The above computed quantity $\bar{u}$ is referred to as the 2-D average concentration. This together with the free boundary variable $d^{\varepsilon}$ constitute the 2-D Average.

4.5. Numerical computations. For computations on the microscale model (2.9)-(2.14), we use the arbitrary Lagrangian Eulerian (ALE) method. This method can be used to solve the partial differential equations on a moving domain and is a generalization of Eulerian and Lagrangian descriptions of the free boundaries. We use the ALE method as implemented in the COMSOL Multiphysics package [16] with Laplacian smoothing [8]. We refer to [8] for more details and a survey of the ALE methods. The computations are carried out for different values of $D$ and $\varepsilon$. The solution of the full 2-D model (microscopic model (2.9)-(2.14)) is approximated by a backward differentiation formula time stepping combined with a finite element method. Further, the (numerical) solutions of the different upscaled models, namely, Hyperbolic (4.1), Simple Averaging (4.2), Upscaled (4.3) described above (the concentration $u_{e}$ and the free boundary variable $d_{e}$ ), are compared at certain times with the transversal average of the concentration $\bar{u}(4.4)$, as well as the free boundary variable $d^{\varepsilon}$ for the 2 -D strip.

The numerical computations for the 2-D strip are assuming an initial equilibrium situation, meaning that no precipitation or dissolution is encountered. In the specific situation considered here, the equilibrium is achieved for $u=0.5$ when $r_{p}=\frac{1}{2} H(v)$, meaning that the precipitation and the dissolution rates are equal. This situation is perturbed by imposing the concentration 0 at the inlet. We also assume that initially the system includes a deposition layer of thickness $d_{I}$ at the lateral boundaries. The 
inlet flow profile is assumed parabolic, and the total water flux into the system is $2 \varepsilon\left(1-d_{I}\right) \bar{q}_{e}$.

Since the fluid flowing in has a low concentration of solute, dissolution will take place at the lateral boundary beginning from the inlet boundary side. Also, the strip becomes wider as the dissolution proceeds. Starting with a width $2 \varepsilon\left(1-d_{I}\right)$, after having dissolved the entire precipitate the strip becomes $2 \varepsilon$ wide.

For the numerical experiments, we take the values

$$
\bar{q}_{e}=1 ; L=1 ; k=1 ; \rho=1,
$$

whereas the initial solute concentration and layer thickness are

$$
u(x, 0)=0.5 \text { and } d(x, 0)=d_{I}=0.2 .
$$

We consider four situations. First we show that all three upscaled models agree well when $\varepsilon$ tends to 0 . Second, we fix $\varepsilon, \bar{q}_{e}$, and $D$ and vary $\alpha$, which basically means varying the diffusion $\varepsilon^{\alpha} D$. This provides information regarding the differences in results of different upscaled models with respect to variations in the value of $\alpha$. Accordingly, it can be used to make informed choices for the upscaled equations for given parameters of $\alpha, \varepsilon, \bar{q}_{e}$, and $D$. Next we fix $D, \bar{q}_{e}$, and $\alpha$ and vary $\varepsilon$ to study the comparisons of the upscaled models with the 2-D strip. Finally we provide the comparison with the fixed geometry and the variable geometry upscaled equations and show that the choice of the appropriate upscaled models is dictated essentially by $\rho$.

Figure 3 is the snapshot for different times for the dissolution process taking place in a thin half-strip. Close to the beginning, at $t=0.02$, the strip has almost uniform concentration in the strip, and the dissolution process starts taking place
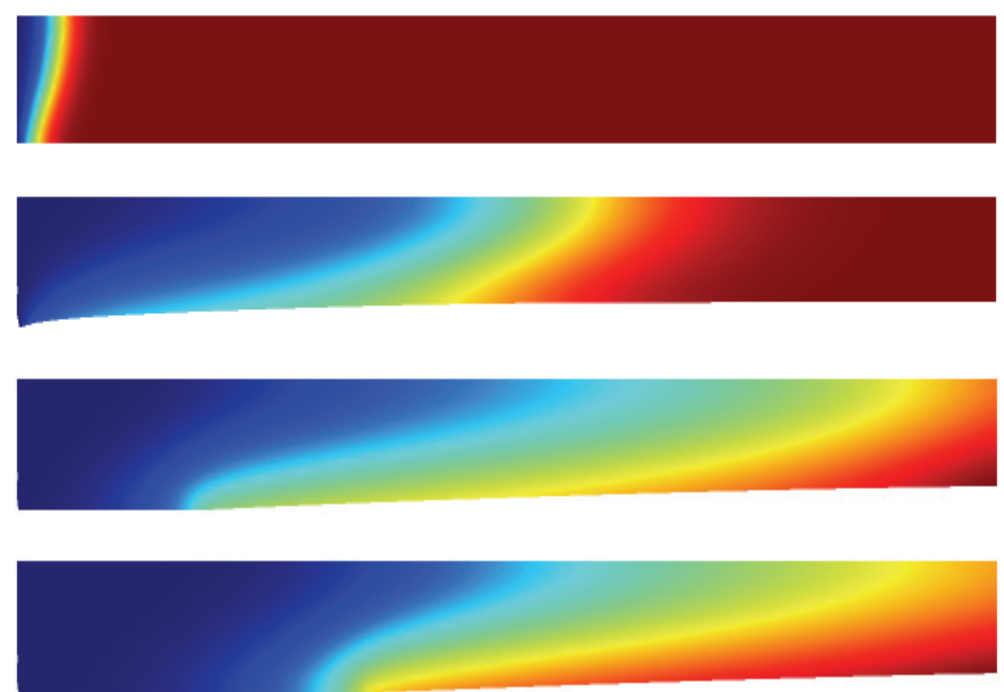

FIG. 3. Time snapshots showing the dissolution process taking place in the thin strip at different times; the top figure is at $t=0.02$, and the bottom one is at $t=1$. The parameter values are $\varepsilon=0.1, D=0.3, \alpha=1.5, \bar{q}_{e}=1$, and $d(x, 0)=0.2$. Note that the dissolution process starts at the left end and that the width of the strip increases gradually as the dissolution front is moving to the right. The initial thickness of the strip is 0.08 , and the final thickness after the dissolution process has finished is 0.1 . 
from the left end $(x=0, y=d-\varepsilon)$; this process continues until the entire precipitate is dissolved. At $y=0$ symmetry conditions are used; therefore no changes in the geometry are encountered there.

Figures 4 and 5 illustrate the convergence of the different upscaled models Simple Averaging (4.2) and Upscaled (4.3) to the hyperbolic model (4.1) as $\varepsilon \rightarrow 0$.
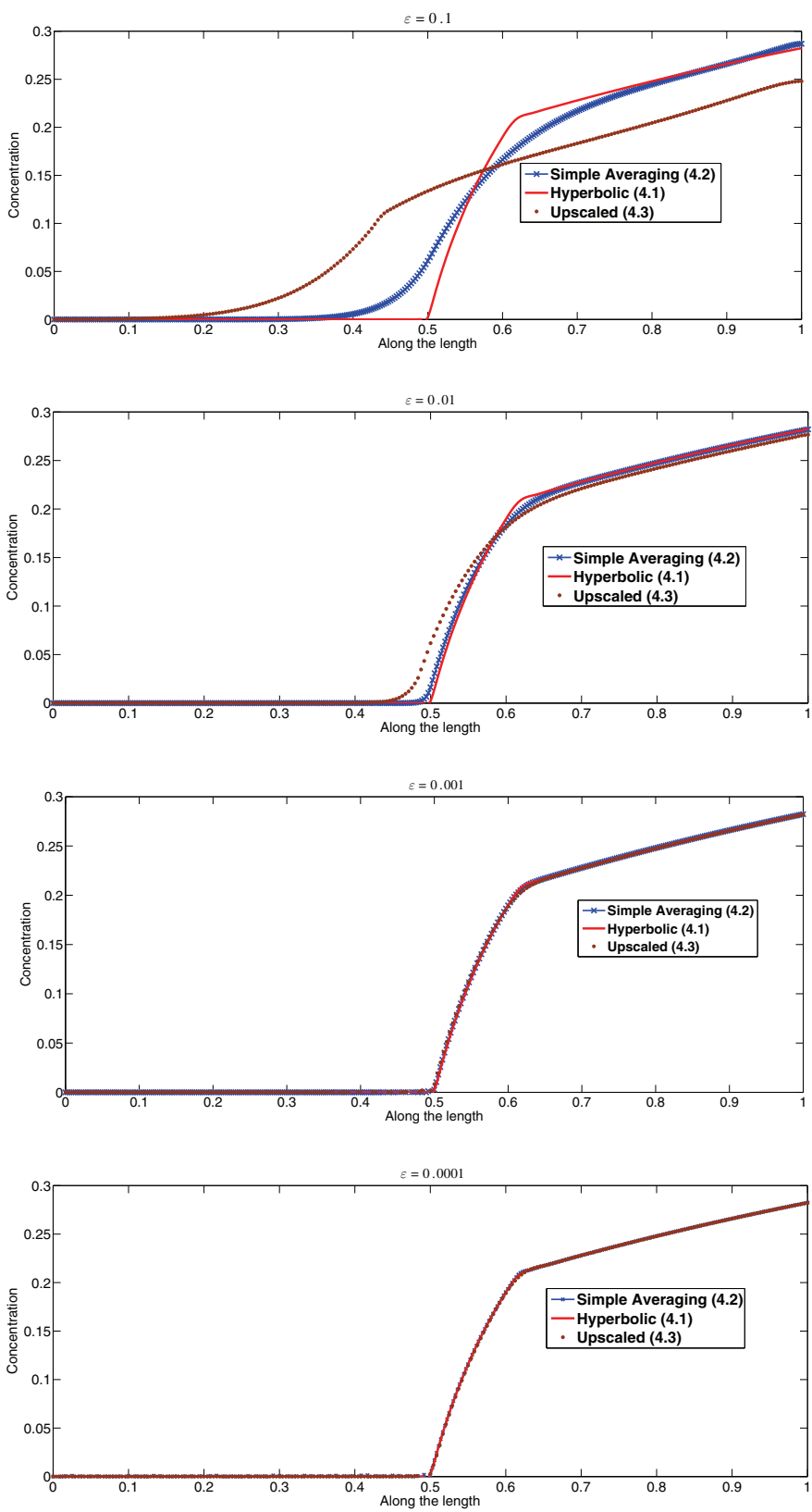

FIG. 4. Convergence of concentration profiles using different upscaled models for varying $\varepsilon=$ $0.1,0.01,0.001,0.0001, D=0.5, \quad \alpha=1, \quad \bar{q}=1$.

Copyright $@$ by SIAM. Unauthorized reproduction of this article is prohibited. 

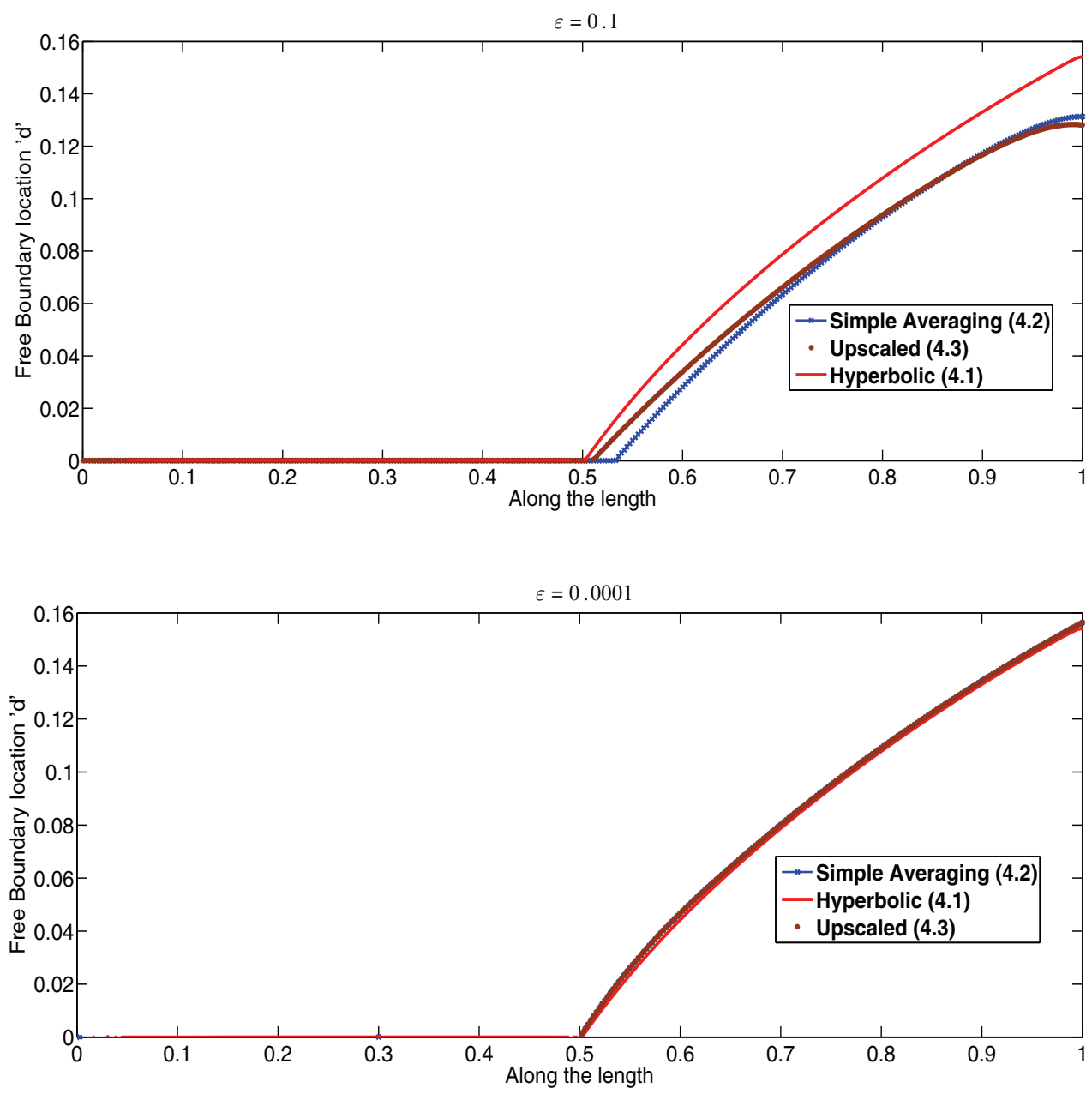

FIG. 5. Convergence of free boundary location d profiles using different upscaled models for varying $\varepsilon=0.1,0.0001, D=0.5, \quad \alpha=1, \quad \bar{q}_{e}=1$.

Letting $\varepsilon$ decrease, it is easy to check formally that all upscaled models reduce to the hyperbolic model.

Further, the numerical results for $\alpha=1$ and $\alpha=1.5$ are compared in Figures 6 (presenting the concentration profiles) and 7 , where the free boundary variable $d$ is presented. As can be clearly observed, the effective model derived in section 3.2 performs better than the simple averaging or the hyperbolic model.

For $\varepsilon=0.01$, the 2-D averaged concentration profile is compared to the effective concentrations in Figure 8. Similarly, in Figure 7 the corresponding free boundaries $d$ are compared. Again the effective model derived here outperforms the other upscaled models.

4.6. Fixed geometry versus variable geometry. When the changes in the geometry on the pore scale are ignored, we refer to it as the fixed geometry case. The upscaling for the fixed geometry as mentioned in Remark 3.1 above leads to the following system of equations: 

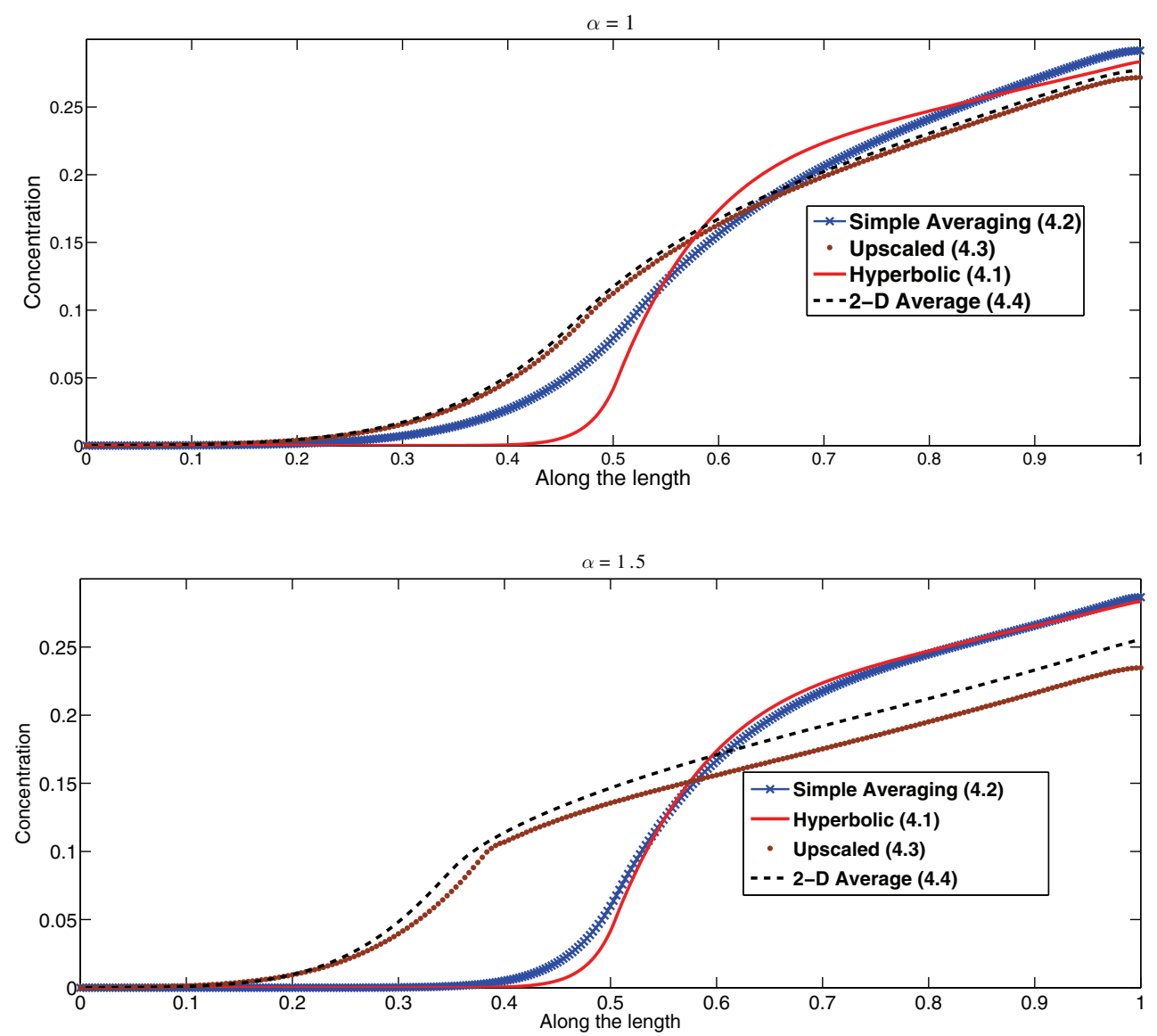

FIG. 6. Comparison of concentration profiles using different upscaled models for varying $\alpha, D=$ $0.3, \varepsilon=0.1, \quad \bar{q}_{e}=1$.

\section{(Fixed Geometry)}

$$
\begin{aligned}
\partial_{t}\left(u_{e}+v_{e}\right) & =\partial_{x}\left\{-u_{e} \bar{q}_{e}+\varepsilon D\left(1+\frac{2 \bar{q}_{e}^{2}}{105 D^{2}}\right) \partial_{x} u_{e}-\varepsilon \frac{1}{15} \frac{\bar{q}_{e}}{D} f\left(u_{e}, v_{e}\right)\right\} \\
\partial_{t} v_{e} & =f\left(u_{e}, v_{e}\right)+\varepsilon\left(-\frac{1}{3 D} \partial_{t} v_{e}+\frac{1}{15 D} \bar{q}_{e} \partial_{x} u_{e}\right) \partial_{1} f\left(u_{e}, v_{e}\right) .
\end{aligned}
$$

To understand when it becomes important to take into account the variable geometry, we compare the solutions of the Upscaled (4.3) model (for $\alpha=1$ ) with the solutions of the (4.5) for different values of $\rho$ (density of salt in the crystal). In Figures 9 and 10 we compare the concentration and the free boundary for the upscaled models obtained in the fixed, respectively, variable geometry case. We see that as $\rho=10$, the solutions do not differ much, whereas for the $\rho=1$ case clearly indicates that the changes in the geometry cannot be ignored and must be taken into account. We see that as $\rho$ increases, the difference between the two models reduces. This is consistent with the observation that in the limit $\rho \searrow \infty$, the variable geometry case reduces to the fixed 

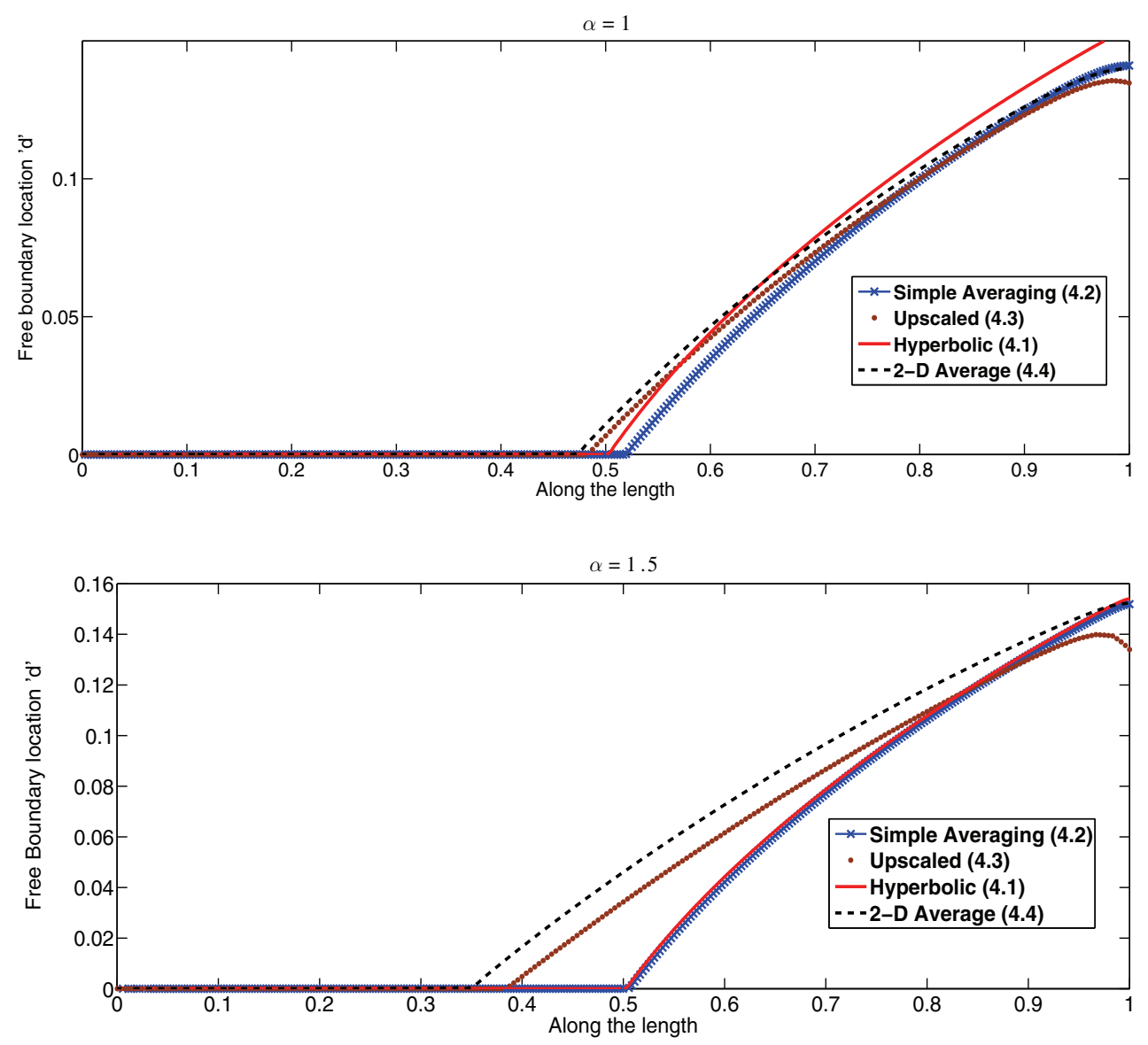

FIG. 7. Comparison of free boundary variable $d$ using different upscaled models for varying $\alpha, D=$ $0.3, \varepsilon=0.1, \quad \bar{q}_{e}=1$.

geometry case. This comparison provides us a useful criterion to decide if the changes in the pore geometry can be ignored.

5. Conclusions and outlook. In this paper, we have derived an upscaled model for the reactive flow in a thin strip in the case of a dominating convective transport. For simplicity, the model is presented in the case of a simple, 2- $\mathrm{D}$ domain (a strip) but takes into account the changes in the microscale geometry that are due to the reactions taking place at the lateral boundaries (the pore walls). The effective model involves a dispersion term that is similar to the Taylor dispersion, whereas the transport is enhanced by reactions.

In deriving the effective equations, we use formal asymptotic methods. The theoretical derivation is sustained by numerical computations, which are carried out in different cases. In all cases the results provided by the upscaled model derived here are compared with the ones obtained by other, simpler upscaled models and with the (transversal) average of the solution to the full problem. Specifically, the following situations are considered: 

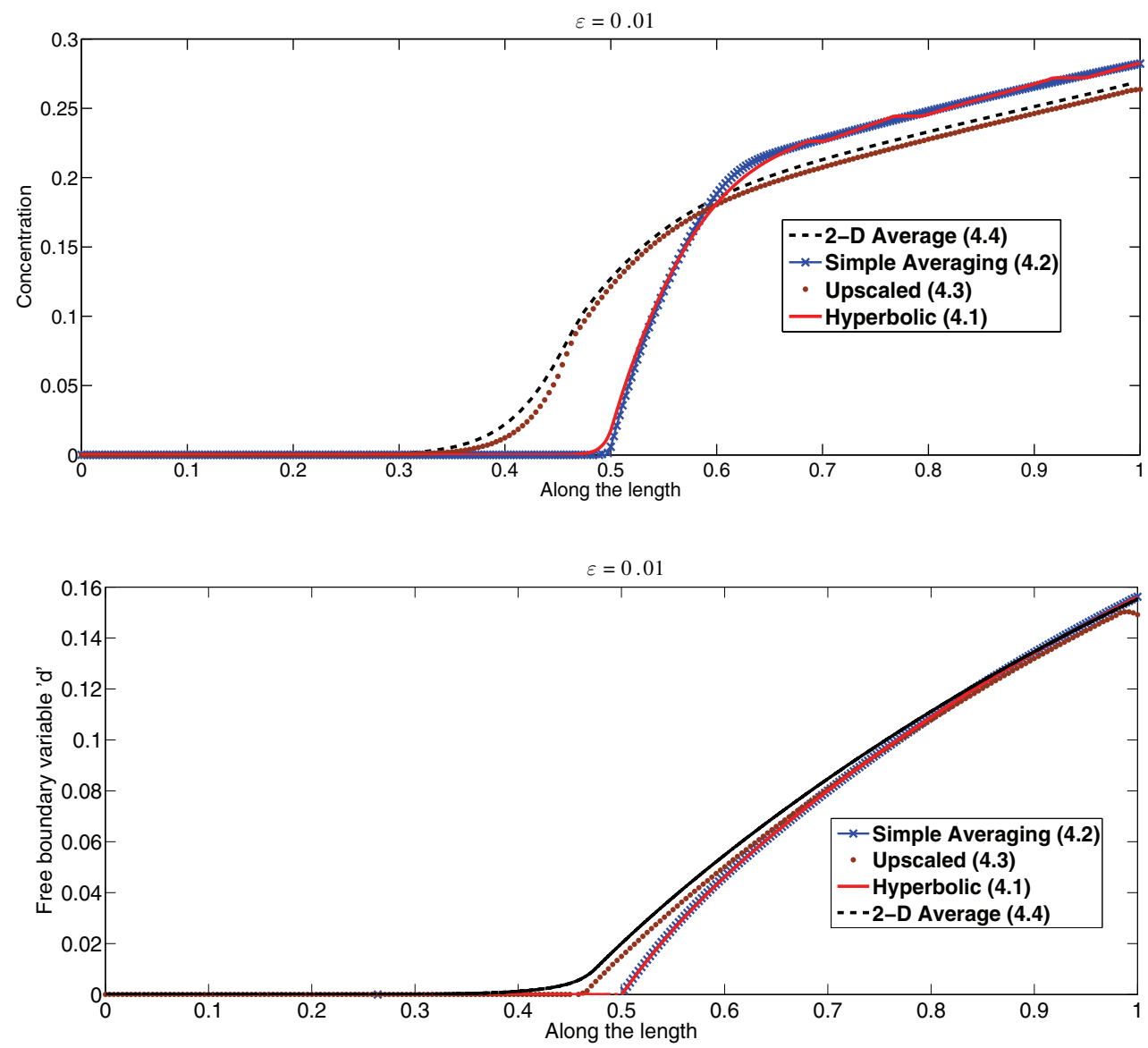

FIG. 8. Comparison of concentration profile and the free boundary variable $d$ using different upscaled models for $\varepsilon=0.01, D=0.3, \quad \alpha=1.5, \quad \bar{q}_{e}=1 ;$ to compare with $\varepsilon=0.1$, see Figures 6 (bottom) and 7 (bottom).

- $\epsilon$, the ratio of the width of the strip and its length, is moderate or small.

- $P e$, the ratio of the diffusive time scale and of the convective one, is of order $\varepsilon^{-1}$ or larger (e.g., $\left.\varepsilon^{-1.5}\right)$.

- $\rho$, the ratio of the density of an element in the adsorbed substance and the solute density is moderate or high.

The following conclusions can be drawn:

- The effective model derived here provides results in excellent agreement with the averaged solutions of the original problem.

- If $\varepsilon$ is moderately small, the present upscaling outperforms other (simpler) models; if $\varepsilon$ becomes very small, all models provide similar results. Therefore, the present approach is recommended in intermediate regimes.

- The upscaling strategy works for $P e=O\left(\varepsilon^{-\alpha}\right)$ whenever $\alpha \in(0,2)$. The present model clearly outperforms simpler effective models as $\alpha$ is increasing.

- Taking into account the changes in the pore geometry is justified, especially for moderate values of $\rho$. In this case, any quantity of adsorbed material leads to changes in the void space that cannot be neglected. As $\rho$ increases, the 

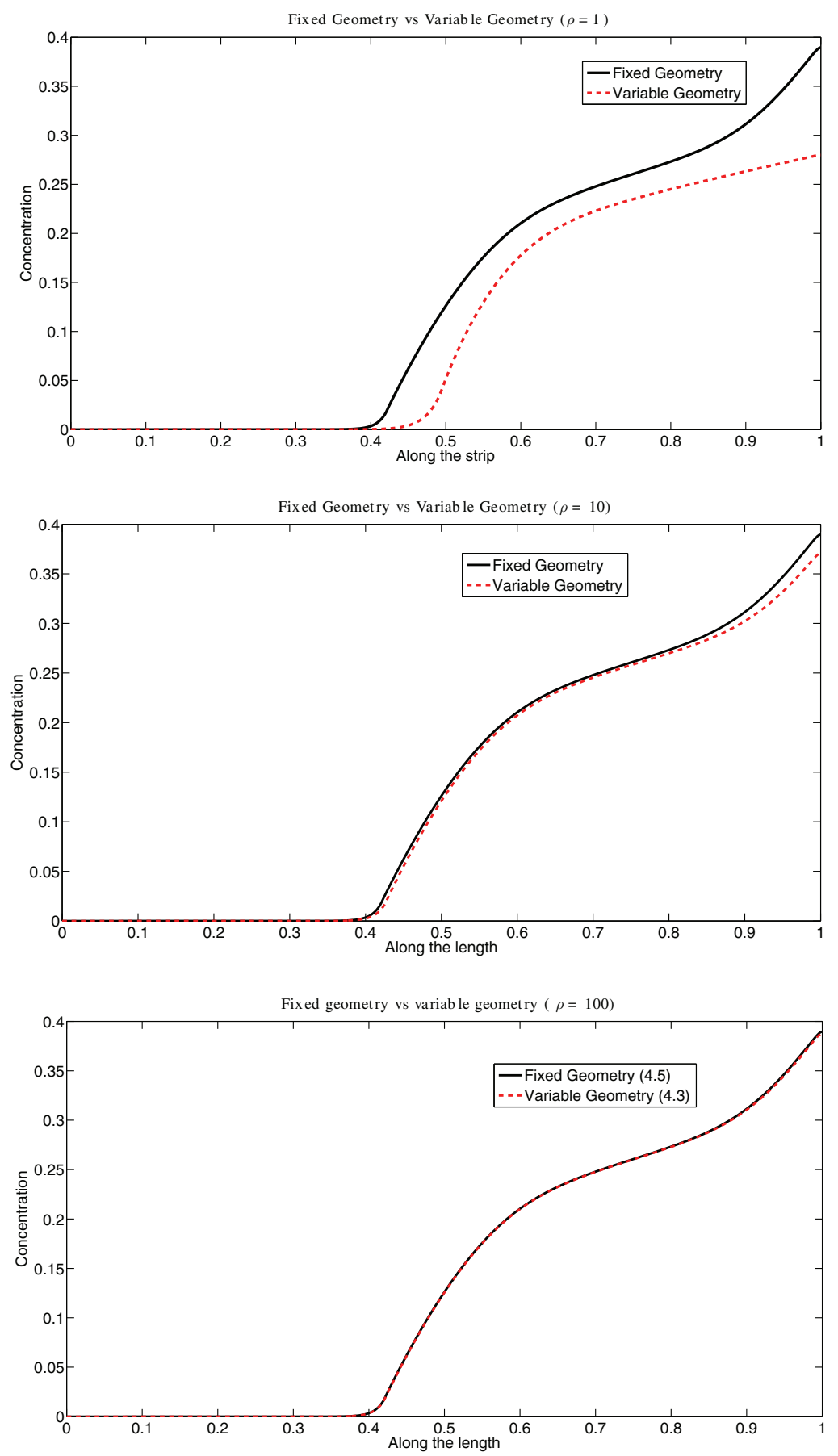

FIG. 9. Comparison of concentration profiles using upscaled models for variable geometry and fixed geometry, $D=0.3, \quad \alpha=1, \quad \bar{q}_{e}=1$.

Copyright $@$ by SIAM. Unauthorized reproduction of this article is prohibited. 

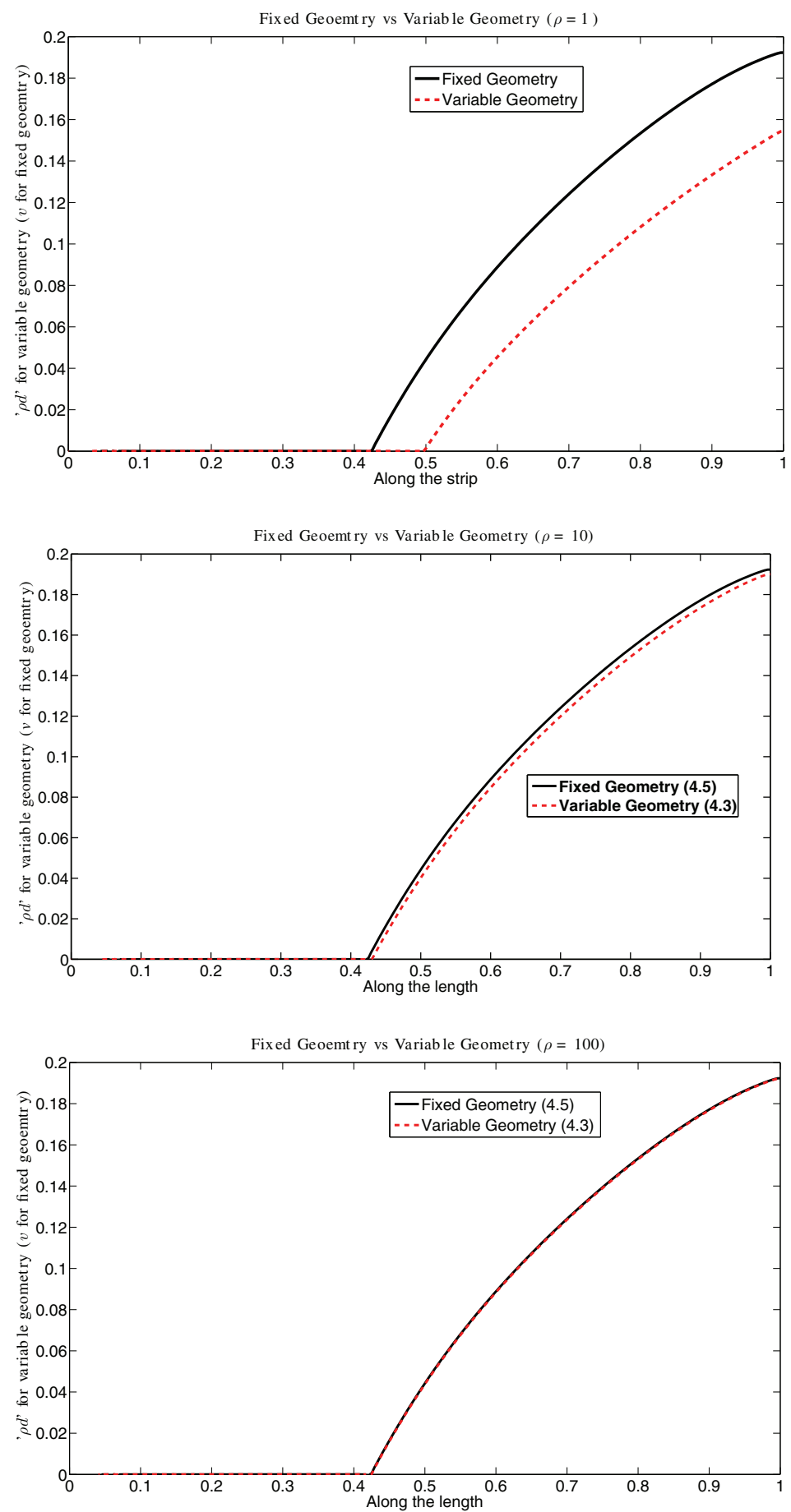

FIG. 10. Comparison of $\rho d$ for variable geometry and $v$ for the fixed geometry, $D=0.3, \quad \alpha=$ $1, \bar{q}_{e}=1$.

Copyright (c) by SIAM. Unauthorized reproduction of this article is prohibited. 
differences between the effective solutions obtained in variable, respectively, fixed geometries are vanishing.

The particular pore structure considered here, a thin strip, may be seen as a representative but simplified pore geometry of a porous medium. However, applying a similar upscaling procedure as here but for a realistic porous media remains a challenging task. Furthermore, giving mathematically rigorous estimates of the errors involved in this upscaling process remains an open question.

Appendix A. Dispersion for the fixed geometry case. Here we give a derivation of the upscaled equations for the case when the pore-scale geometry does not change as a result of reactions taking place. The derivation differs slightly from the one in [10], where the same situation is considered. While the cited work uses anisotropic singular perturbation technique [31] to obtain the upscaled equations, here the formal homogenization techniques have been used. As will be seen below, the results agree well.

The 2-D bounded domain representing the strip is given by

$$
\Omega_{\varepsilon}:=\{(x, y) \in \mathbb{R} \mid 0<x<1,-\varepsilon \leq y \leq \varepsilon\} .
$$

The boundaries of $\Omega_{\varepsilon}$ are then defined by the lateral boundary $\Gamma_{\varepsilon}$, given by

$$
\Gamma_{\varepsilon}:=\{(x, y) \mid 0 \leq x \leq 1, y \in\{-\varepsilon, \varepsilon\}\},
$$

the inlet boundary $\Gamma_{i}$,

$$
\Gamma_{i}:=\{(x, y) \mid x=0,-\varepsilon \leq y \leq \varepsilon\}
$$

and the outflow boundary $\Gamma_{o}$,

$$
\Gamma_{o}:=\{(x, y) \mid x=1,-\varepsilon \leq y \leq \varepsilon\} .
$$

For modeling the crystal precipitation/dissolution, we consider the following dimensionless system of equations that describes the flow of the solutes in a fixed geometry.

$$
\begin{aligned}
u_{t}^{\varepsilon} & =\nabla \cdot\left(\varepsilon D \nabla u^{\varepsilon}-q^{\varepsilon} u^{\varepsilon}\right) \text { in } \Omega_{\varepsilon}, \\
\varepsilon^{2} \mu \triangle q^{\varepsilon} & =\nabla p^{\varepsilon} \quad \text { in } \Omega_{\varepsilon}, \\
\nabla \cdot q^{\varepsilon} & =0 \text { in } \Omega_{\varepsilon} \\
v_{t}^{\varepsilon} & =\varepsilon f\left(u^{\varepsilon}, v^{\varepsilon}\right) \text { on } \Gamma_{\varepsilon}, \\
-\nu^{\varepsilon} \cdot \varepsilon D \nabla u^{\varepsilon} & =v_{t}^{\varepsilon} \text { on } \Gamma_{\varepsilon}, \\
q^{\varepsilon} & =0 \text { on } \Gamma_{\varepsilon} \\
u^{\varepsilon} & =u_{b_{i}} \text { on } \Gamma_{i}, \\
q^{\varepsilon} & =q_{b_{i}} \text { on } \Gamma_{i}, \\
\nu \cdot \nabla u^{\varepsilon} & =0 \text { on } \Gamma_{o}, \\
q^{\varepsilon} & =q_{b_{0}} \text { on } \Gamma_{o}, \\
u^{\varepsilon}(x, y, 0) & =u_{0}(x, y), \\
v^{\varepsilon}(x, 0) & =v_{0}(x) .
\end{aligned}
$$

Recall that the pore geometry is fixed, and so, to take into account the thickness of the deposition, another variable $v$ is needed to account for the reactant/product 
on the lateral boundaries. After rescaling $x:=x ; y=y / \varepsilon$, we obtain the following, considering only equations which are relevant to the present discussion:

$\varepsilon^{2} \mu\left(\partial_{x x} q^{(1) \varepsilon}+\varepsilon^{-2} \partial_{y y} q^{(1) \varepsilon}, \partial_{x x} q^{(2) \varepsilon}, \varepsilon^{-2} \partial_{y y} q^{(2) \varepsilon}\right)^{T}=\left(\partial_{x} p^{\varepsilon}, \varepsilon^{-1} \partial_{y} p^{\varepsilon}\right)^{T} \quad$ in $\quad \Omega_{\varepsilon}$,

$$
\begin{aligned}
\partial_{x} q^{(1) \varepsilon}+\varepsilon^{-1} \partial_{y} q^{(2) \varepsilon} & =0 \text { in } \Omega_{\varepsilon}, \\
v_{t}^{\varepsilon} & =f\left(u^{\varepsilon}, v^{\varepsilon}\right) \text { on } \Gamma_{\varepsilon}, \\
-\nu^{\varepsilon} \cdot \varepsilon D\left(\partial_{x} u^{\varepsilon}, \varepsilon^{-1} \partial_{y} u^{\varepsilon}\right) & =\varepsilon v_{t}^{\varepsilon} \text { on } \Gamma_{\varepsilon}, \\
q^{\varepsilon} & =0 \text { on } \Gamma_{\varepsilon} .
\end{aligned}
$$

Since the geometry is assumed to be fixed, we can solve the Stokes equation (A.2)(A.3) with the no-slip boundary condition (A.6) if we assume a parabolic inlet flow profile (Poiseuille flow)

$$
q^{(1) \varepsilon}(y)=Q\left(1-y^{2}\right), \quad q^{(2) \varepsilon}=0,
$$

where $Q>0$ is a given constant, depending upon the pressure gradient.

We assume the following asymptotic expansion for $u^{\varepsilon}$ and $v^{\varepsilon}$ :

$$
\begin{aligned}
& u^{\varepsilon}=u_{0}+\varepsilon u_{1}+O\left(\varepsilon^{2}\right), \\
& v^{\varepsilon}=v_{0}+\varepsilon v_{1}+O\left(\varepsilon^{2}\right) .
\end{aligned}
$$

Using the expansion above in the convection-diffusion equation (A.1) with boundary condition (A.5), we obtain

$$
\begin{aligned}
\partial_{t} u_{0}+\varepsilon \partial_{t} u_{1}= & \varepsilon D \partial_{x x} u_{0}+\varepsilon^{-1} D \partial_{y y} u_{0}+\varepsilon^{2} D \partial_{x x} u_{1}+D \partial_{y y} u_{1} \\
& -Q\left(1-y^{2}\right) \partial_{x}\left(u_{0}+\varepsilon u_{1}\right)+O\left(\varepsilon^{2}\right) \text { in } \Omega_{\varepsilon}, \\
D\left(\partial_{y} u_{0}+\varepsilon \partial_{y} u_{1}\right)= & \varepsilon \partial_{t} v_{0}+\varepsilon^{2} \partial_{t} v_{1}+O\left(\varepsilon^{3}\right) \quad \text { on } \Gamma_{\varepsilon},
\end{aligned}
$$

The $\varepsilon^{-1}$ term is

$$
\begin{aligned}
D \partial_{y y} u_{0} & =0, \\
D \partial_{y} u_{0} & =0 \text { at } y=\{-1,1\},
\end{aligned}
$$

and hence we conclude

$$
u_{0}(x, y, t)=u_{0}(x, t)
$$

The $\varepsilon^{0}$ term is

$$
\begin{aligned}
\partial_{t} u_{0}-D \partial_{y y} u_{1}+Q\left(1-y^{2}\right) \partial_{x} u_{0} & =0, \\
-D \partial_{y} u_{1} & =\partial_{t} v_{0} \quad \text { at } y=1, \\
\partial_{y} u_{1} & =0 \quad \text { at } y=0 .
\end{aligned}
$$

Integrating the first equation from $y=0$ to 1 and using the boundary conditions (A.10) and (A.11) gives

$$
\partial_{t} u_{0}+\partial_{t} v_{0}+\frac{2}{3} Q \partial_{x} u_{0}=0
$$

Copyright $@$ by SIAM. Unauthorized reproduction of this article is prohibited. 
To obtain an expression for $u_{1}$, we eliminate $\partial_{t} u_{0}$ from (A.9). Subtracting (A.12) from (A.9) gives

$$
-D \partial_{y y} u_{1}+Q\left(\frac{1}{3}-y^{2}\right) \partial_{x} u_{0}-\partial_{t} v_{0}=0
$$

Successively integrating (A.13) with respect to $y$, we get for $u_{1}(x, y, t)$ as

$$
u_{1}(x, y, t)=\frac{Q}{D}\left(\frac{y^{2}}{6}-\frac{y^{4}}{12}+C_{0}(x, t)\right) \partial_{x} u_{0}+\frac{1}{D}\left(\frac{-y^{2}}{2}+C_{1}(x, t)\right) \partial_{t} v_{0}
$$

where $C_{0}, C_{1}$ are constants of integration. As will be seen later, there is no need for specifying the expressions for $C_{0}, C_{1}$ since their effects get canceled in the averaging process.

The $\varepsilon^{1}$ term is

$$
\begin{aligned}
\partial_{t} u_{1}-D\left(\partial_{x x} u_{0}+\partial_{y y} u_{2}\right)+Q\left(1-y^{2}\right) \partial_{x} u_{1} & =0 \\
-D \partial_{y} u_{2} & =\partial_{t} v_{1} .
\end{aligned}
$$

Integrating (A.15) from $y=0$ to $y=1$, we obtain

$$
\partial_{t} \int_{0}^{1} u_{1}-D \partial_{x x} u_{0}+k \partial_{t} v_{1}+Q \int_{0}^{1}\left(1-y^{2}\right) \partial_{x} u_{1} d y=0
$$

Define

$$
\bar{u}_{1}=\int_{0}^{1} u_{1} d y
$$

and with this definition, (A.17) becomes

$$
\partial_{t} \bar{u}_{1}-D \partial_{x x} u_{0}+\partial_{t} v_{1}+Q \partial_{x} \bar{u}_{1}=Q \int_{0}^{1} y^{2} \partial_{x} u_{1} d y
$$

Adding (A.18) and (A.12) gives

$$
\begin{aligned}
\partial_{t}\left(u_{0}+\varepsilon \bar{u}_{1}\right)- & \varepsilon D \partial_{x x} u_{0}+\partial_{t}\left(v_{0}+\varepsilon v_{1}\right)+\frac{2}{3} Q \partial_{x}\left(u_{0}+\varepsilon \bar{u}_{1}\right) \\
= & \varepsilon Q\left(\int_{0}^{1} y^{2} \partial_{x} \bar{u}_{1} d y-\frac{1}{3} \int_{0}^{1} \partial_{x} \bar{u}_{1} d y\right) .
\end{aligned}
$$

Define

$$
\begin{aligned}
& u_{e}=u_{0}+\varepsilon \bar{u}_{1}, \\
& v_{e}=v_{0}+\varepsilon v_{1} .
\end{aligned}
$$

With this definition, (A.19) becomes

$$
\partial_{t}\left(u_{e}+v_{e}\right)-\varepsilon D \partial_{x x} u_{e}+\frac{2}{3} Q \partial_{x} u_{e}=-\varepsilon^{2} D \partial_{x x} \bar{u}_{1}+\varepsilon Q \int_{0}^{1}\left(y^{2}-\frac{1}{3}\right) \partial_{x} \bar{u}_{1} .
$$

To compute the $\int_{0}^{1}\left(y^{2}-\frac{1}{3}\right) \partial_{x} \bar{u}_{1}$, we can use the expression for $u_{1}$ as obtained in (A.14). Basically we need to compute the integrals

$$
\int_{0}^{1}\left(y^{2}-\frac{1}{3}\right)\left(\frac{y^{2}}{6}-\frac{y^{4}}{12}+C_{0}\right) d y=\frac{8}{945}
$$

Copyright (c) by SIAM. Unauthorized reproduction of this article is prohibited. 
and

$$
\int_{0}^{1}\left(y^{2}-\frac{1}{3}\right)\left(C_{1}-\frac{y^{2}}{2}\right) d y=\frac{-2}{45} .
$$

Here it is to be noted that the precise expressions for $C_{0}, C_{1}$ are of no consequence. Substituting the value of $\int_{0}^{1}\left(y^{2}-\frac{1}{3}\right) \partial_{x} \bar{u}_{1}$, using the integrals computed above, in (A.20) gives

$$
\partial_{t}\left(u_{e}+v_{e}\right)-\varepsilon D \partial_{x x} u_{e}+\frac{2 Q}{3} \partial_{x} u_{e}=-\varepsilon^{2} D \partial_{x x} \bar{u}_{1}+\frac{8 \varepsilon}{945} \frac{Q^{2}}{D} \partial_{x x} u_{0}-\frac{\varepsilon Q}{D} \frac{2}{45} \partial_{x t} v_{0} .
$$

Hence the upscaled equation takes the form

$$
\begin{aligned}
\partial_{t}\left(u_{e}+v_{e}\right)-\varepsilon D \partial_{x x} u_{e}+\frac{2 Q}{3} \partial_{x} u_{e}= & -\varepsilon^{2} D \partial_{x x} \bar{u}_{1} \\
& +\frac{8 \varepsilon}{945} \frac{Q^{2}}{D} \partial_{x x} u_{0}+\varepsilon^{2} \frac{8}{945} \frac{Q^{2}}{D} \partial_{x x} \bar{u}_{1} \\
& -\varepsilon^{2} \frac{8}{945} \frac{Q^{2}}{D} \partial_{x x} \bar{u}_{1}-\frac{\varepsilon Q}{D} \frac{2}{45} \partial_{x t} v_{0} \\
& -\varepsilon^{2} \frac{Q}{D} \frac{2}{45} \partial_{x t} v_{1}+\varepsilon^{2} \frac{Q}{D} \frac{2}{45} \partial_{x t} v_{1} .
\end{aligned}
$$

This can then be rewritten up to an error of order $O\left(\varepsilon^{2}\right)$ as

$$
\begin{aligned}
\partial_{t}\left(u_{e}+v_{e}\right)-\varepsilon D \partial_{x x} u_{e}+\frac{2 Q}{3} \partial_{x} u_{e}= & -\varepsilon^{2} D \partial_{x x} \bar{u}_{1} \\
& +\frac{8 \varepsilon}{945} \frac{Q^{2}}{D}\left(\partial_{x x}\left(u_{0}+\varepsilon \bar{u}_{1}\right)\right) \\
& -\varepsilon^{2} \frac{8}{945} \frac{Q^{2}}{D} \partial_{x x} u_{1}-\varepsilon \frac{Q}{D} \frac{2}{45}\left(\partial_{x t}\left(v_{0}+\varepsilon v_{1}\right)\right) \\
& +\varepsilon^{2} \frac{Q}{D} \frac{2}{45} \partial_{x t} v_{1} .
\end{aligned}
$$

For $v_{e}$, we can have, formally, by using the Taylor expansion of $f\left(u^{\varepsilon}, v^{\varepsilon}\right)$ around $\left(u_{e}, v_{e}\right)$,

$$
\begin{aligned}
\partial_{t} v_{e} & =f\left(u^{\varepsilon}, v^{\varepsilon}\right) \\
& =f\left(u_{0}+\varepsilon \bar{u}_{1}, v_{0}+\varepsilon v_{1}\right)+\varepsilon\left(\left.u_{1}\right|_{y=1}-\bar{u}_{1}\right) \partial_{1} f\left(u_{0}+\varepsilon \bar{u}_{1}, v_{0}+\varepsilon v_{1}\right)+O\left(\varepsilon^{2}\right) \\
& =f\left(u_{e}, v_{e}\right)+\varepsilon\left(\left.u_{1}\right|_{y=1}-\bar{u}_{1}\right) \partial_{1} f\left(u_{e}, v_{e}\right)+O\left(\varepsilon^{2}\right)
\end{aligned}
$$

And from the expression for $u_{1}$ using (A.14), we have

$$
\begin{aligned}
u_{1}(y=1)-\bar{u}_{1} & =\frac{Q}{D}\left(\frac{1}{12}+C_{0}-\frac{7}{180}-C_{0}\right) \partial_{x} u_{0}+\frac{1}{D}\left(C_{1}-\frac{1}{2}-C_{1}+\frac{1}{6}\right) \\
& =\left\{\frac{Q}{D} \frac{2}{45} \partial_{x} u_{0}-\frac{1}{3} \frac{1}{D} \partial_{t} v_{0}\right\} .
\end{aligned}
$$

Once again, we note that the values of $C_{0}, C_{1}$ are immaterial. (A.22) and (A.23) give us the final expression for $v_{e}$,

$$
\partial_{t} v_{e}=f\left(u_{e}, v_{e}\right)+\varepsilon\left\{\frac{Q}{D} \frac{2}{45} \partial_{x} u_{0}-\frac{1}{3} \frac{1}{D} \partial_{t} v_{0}\right\} \partial_{1} f\left(u_{e}, v_{e}\right)
$$

Copyright (c) by SIAM. Unauthorized reproduction of this article is prohibited. 
To summarize, we obtain the set of effective equations for the fixed geometry case up to $O\left(\varepsilon^{2}\right)$,

$$
\begin{gathered}
\partial_{t}\left(u_{e}+v_{e}\right)=\partial_{x}\left\{-u_{e} \bar{q}_{e}+\varepsilon D\left(1+\frac{2 \bar{q}_{e}^{2}}{105 D^{2}}\right) \partial_{x} u_{e}-\varepsilon \frac{1}{15} \frac{\bar{q}_{e}}{D} f\left(u_{e}, v_{e}\right)\right\}, \\
\partial_{t} v_{e}=f\left(u_{e}, v_{e}\right)+\varepsilon\left(-\frac{1}{3 D} \partial_{t} v_{e}+\frac{1}{15 D} \bar{q}_{e} \partial_{x} u_{e}\right) \partial_{1} f\left(u_{e}, v_{e}\right) .
\end{gathered}
$$

Acknowledgments. The authors are members of the International Research Training Group, Nonlinear Upscaling in Porous Media (NUPUS), Stuttgart. The authors would like to thank Mark Peletier, Andro Mikelić, and Peter Notten for their useful comments and discussions. Last, but not the least, the authors express their appreciations and thanks to the anonymous referees for their useful suggestions.

\section{REFERENCES}

[1] G. Allaire, R. Brizzi, And A. Mikelić, Two-scale expansion with drift approach to the Taylor dispersion for reactive transport through porous media, Chem. Engrg. Sci., 65 (2010), pp. 2292-2300.

[2] J. L. Auriault And P. M. Adler, Dispersion in porous media: By multiple scale expansions, Adv. Water Resour., 18 (1995), pp. 211-226.

[3] J. Bear, Dynamics of Fluids in Porous Media, Dover, New York, 1988.

[4] C. W. J. Berentsen, M. L. Verlaan, and C. P. J. W. V. Kruijsdijk, Upscaling and reversibility of Taylor dispersion in heterogeneous porous media, Phys. Rev. E(3), 71 (2005), Article 046308.

[5] W. Chen, H. J. H. Brouwers, And Z. H. Shui, Three-dimensional computer modeling of slag cement hydration, J. Mater. Sci., 42 (2007), pp. 9595-9610.

[6] C. Choquet And A. Mikelić, Laplace transform approach to the rigorous upscaling of the infinite adsorption rate reactive flow under dominant Peclet number through a pore, Appl. Anal., 87 (2008), pp. 1373-1395.

[7] C. Conca, J. I. Díaz, and C. Timofte, Effective chemical processes in porous media, Math. Models Methods Appl. Sci., 13 (2003), pp. 1437-1462.

[8] J. Donea, A. Huerta, J.-P. Ponthot, and A. Rodríguez-Ferran, Arbitrary Lagrangian-Eulerian methods, Encyclopedia Comput. Mech., 1 (2004), pp. 413-437.

[9] C. J. V. DuiJn And P. Knabner, Travelling wave behaviour of crystal dissolution in porous media flow, European J. Appl. Math., 8 (1997), pp. 49-72.

[10] C. J. v. Duijn, A. Mikelić, I. S. Pop, AND C. Rosier, Effective dispersion equations for reactive flows with dominant Peclet and Damköhler numbers, Adv. Chem. Engrg., 34 (2008), pp. $1-45$.

[11] C. J. v. DuiJn And I. S. Pop, Crystal dissolution and precipitation in porous media: Pore scale analysis, J. Reine Angew. Math., 577 (2004), pp. 171-211.

[12] J. EBERHARD, Upscaling for stationary transport in heterogeneous porous media, Multiscale Model. Simul., 3 (2005), pp. 957-976.

[13] F. Heese, F. A. Radu, M. Thullner, and S. Attinger, Upscaling of the advection-diffusionreaction equation with monod reaction, Adv. Water Resour., 32 (2009), pp. 1336-1351.

[14] U. Hornung AND W. JäGER, Diffusion, convection, adsorption, and reaction of chemicals in porous media, J. Differential Equations, 92 (1991), pp. 199-225.

[15] O. Iliev, A. Mikelić, And P. Popov, On upscaling certain flows in deformable porous media, Multiscale Model. Simul., 7 (2008), pp. 93-123.

[16] COMSOL Inc., http://www.comsol.com.

[17] A. M. Lankhorst, B. D. Paarhuis, H. J. M. C. Terhorst, P. J. P. M. Simons, and C. R. KLEIJN, Transient ALD simulations for a multi-wafer reactor with trenched wafers, Surface Coatings Technol., 201 (2007), pp. 8842-8848.

[18] A. Mikelić, V. Devigne, And C. J. V. Duijn, Rigorous upscaling of the reactive flow through a pore, under dominant Peclet and Damkohler numbers, SIAM J. Math. Anal., 38 (2006), pp. $1262-1287$.

[19] A. Mikelić And C. J. v. DuiJn, Rigorous derivation of a hyperbolic model for Taylor dispersion, Math. Models Methods Appl. Sci., to appear.

Copyright (c) by SIAM. Unauthorized reproduction of this article is prohibited. 
[20] A. Mikelić And C. Rosier, Rigorous upscaling of the infinite adsorption rate reactive flow under dominant Peclet number through a pore, Ann. Univ. Ferrara Sez. VII Sci. Mat., 53 (2007), pp. 333-359.

[21] A. Muntean AND M. BöHm, Interface conditions for fast-reaction fronts in wet porous mineral materials: The case of concrete carbonation, J. Engrg. Math., 65 (2009), pp. 89-100.

[22] A. Muntean and M. BöHm, A moving-boundary problem for concrete carbonation: Global existence and uniqueness of weak solutions, J. Math. Anal. Appl., 350 (2009), pp. 234-251.

[23] M. Neuss-Radu, Some extensions of two-scale convergence, C. R. Math. Acad. Sci. Paris Sér. I Math., 322 (1996), pp. 899-904.

[24] T. L. V. NoORdEn, Crystal precipitation and dissolution in a porous medium: Effective equations and numerical experiments, Multiscale Model. Simul., 7 (2008), pp. 1220-1236.

[25] T. L. V. Noorden, Crystal precipitation and dissolution in a thin strip, European J. Appl. Math., 20 (2009), pp. 69-91.

[26] T. L. V. Noorden AND I. S. Pop, A Stefan problem modelling dissolution and precipitation, IMA J. Appl. Math., 73 (2008), pp. 393-411.

[27] T. L. V. Noorden, I. S. Pop, A. Ebigbo, And R. Helmig, An upscaled model for biofilm growth in a thin strip, Water Resour. Res., 46 (2010), W06505.

[28] J. F. M. Oudenhoven, K. Kumar, M. H. J. M. D. Croon, T. v. Dongen, M. Mulder, R. A. H. Niessen, I. S. Pop, And P. H. L. Notten, Chemical vapor deposition of TiO2 in trench structures: An experimental and theoretical study, in preparation.

[29] A. RaOof And S. HassanizadeH, Upscaling transport of adsorbing solutes in porous media, J. Porous Media, 13 (2010), pp. 395-408.

[30] A. RiJnks, M. Darwish, and H. Bruining, Computation of the longitudinal dispersion coefficient in an adsorbing porous medium using homogenization, in Proceedings of the COMSOL Conference, Milan, (2009); http://www.comsol.se/academic/papers/6756.

[31] J. Rubinstein and R. MAURI, Dispersion and convection in periodic porous media, SIAM J. Appl. Math., 46 (1986), pp. 1018-1023.

[32] G. I. TAYLOR, Dispersion of soluble matter in solvent flowing slowly through a tube, Proc. R. Soc. Lond. Ser. A Math. Phys. Eng. Sci., 219 (1953), pp. 186-203.

[33] R. P. Tijburg, J. G. M. Ligthart, H. K. Kuiken, and J. J. Kelly, Centrifugal etching, J. Electrochem. Soc., 150 (2003), pp. C440-C444.

[34] C. Vuik And C. Cuvelier, Numerical solution of an etching problem, J. Comput. Physics, 59 (1985), pp. 247-263.

[35] F. Weller, Modeling, Analysis, and Simulation of Thrombosis and Hemostasis, Ph.D. thesis, Universität Heidelberg, Institut für Angewandte Mathematik, Heidelberg, 2008.

[36] B. D. WoOd, K. RAdAKOvich, AND F. Golfier, Effective reaction at a fluidsolid interface: Applications to biotransformation in porous media, Adv. Water Resour., 30 (2007), pp. 1630-1647.

Copyright (c) by SIAM. Unauthorized reproduction of this article is prohibited. 